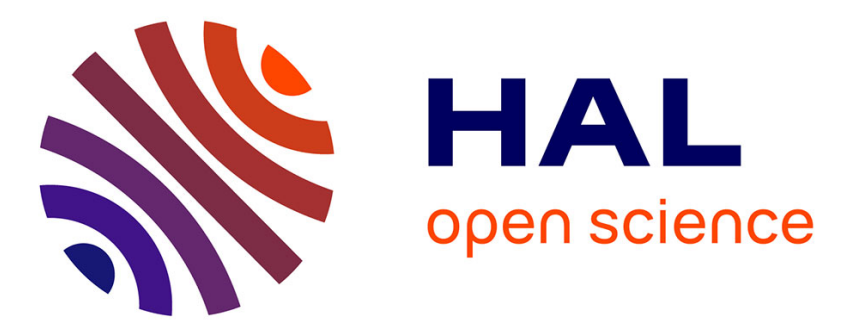

\title{
Squeal analysis based on the effect and determination of the most influential contacts between the different components of an automotive brake system
}

\author{
Enora Denimal, Jean-Jacques Sinou, S. Nacivet, L. Nechak
}

\section{- To cite this version:}

Enora Denimal, Jean-Jacques Sinou, S. Nacivet, L. Nechak. Squeal analysis based on the effect and determination of the most influential contacts between the different components of an automotive brake system. International Journal of Mechanical Sciences, 2019, 151, pp.192-213. 10.1016/j.ijmecsci.2018.10.054 . hal-02957511

HAL Id: hal-02957511

https://hal.science/hal-02957511

Submitted on 11 Jun 2021

HAL is a multi-disciplinary open access archive for the deposit and dissemination of scientific research documents, whether they are published or not. The documents may come from teaching and research institutions in France or abroad, or from public or private research centers.
L'archive ouverte pluridisciplinaire HAL, est destinée au dépôt et à la diffusion de documents scientifiques de niveau recherche, publiés ou non, émanant des établissements d'enseignement et de recherche français ou étrangers, des laboratoires publics ou privés. 
E. Denimal, J-J. Sinou, S. Nacivet, L. Nechak, Squeal analysis based on the effect and determination of the most influential contacts between the different components of an automotive brake system, International Journal of Mechanical Sciences, 151 (2019), 192-213. 10.1016/j.ijmecsci.2018.10.054

\title{
Squeal analysis based on the effect and determination of the most influential contacts between the different components of an automotive brake system
}

\author{
Enora Denimal ${ }^{1,2,}$, Jean-Jacques Sinou ${ }^{1,3,}{ }^{*}$, Samuel Nacivet ${ }^{2}$, and L. Nechak ${ }^{1}$ \\ ${ }^{1}$ Laboratoire de Tribologie et Dynamique des Systèmes, UMR CNRS 5513, École Centrale de Lyon, \\ 36 avenue Guy de Collongue 69134 Écully Cedex, France \\ ${ }^{2}$ PSA Peugeot Citroën, Centre technique de la Garenne Colombes, 18 rue des Fauvelles, \\ 92250 La Garenne Colombes, France \\ ${ }^{3}$ Institut Universitaire de France, 75005 Paris, France \\ ${ }^{*}$ Corresponding authors: enora.denimal@inria.fr ; jean-jacques.sinou@ec-lyon.fr
}

\begin{abstract}
Many numerical simulations have been dedicated to the brake squeal prediction by considering the predominant influence of the frictional interface between the pads lining and the disc. However, many others contact interfaces are present in an automotive brake system. Nowadays, the influence of the state of these contacts on the stability behavior of an automotive brake system and thus, on the squeal propensity is not yet well understood.

This paper attempts to provide a huge investigation on the sensitivity of squeal propensity to the various contact interfaces between pads, piston, bracket and caliper. The main contribution of the present study aims not only to give a better understanding on the role of these numerous contacts in an automotive brake system but also to propose a strategy based on genetics algorithms in order to reduce the number of simulations to achieve by ranking the effects of all the independent contact states in a pre-design process.
\end{abstract}

Keywords Automotive brake system, Complex Eigenvalue Analysis, Finite Element Model, Multi-contacts, Friction, Genetic Algorithm.

\section{Introduction}

Although squeal noise does not affect braking efficiency, the resulting self-excited vibrations and squeal noise of the car disc brakes are a source of considerable discomfort to the user and leads to customer dissatisfaction. Therefore, the growing demands of having quieter brakes and the customer complaints that may result in significant warranty costs motivate the need to study brake squeal early in the design process. Thereby, a better understanding and prediction of the propensity of squeal noise in car brake systems is an important challenge that is faced by the automotive engineers. For example, many studies on Finite Element Models (FEM) for automotive brake squeal have been conducted in recent years [1-8]. Different methodologies are available to predict such phenomenon and two main categories are distinguishable: the dynamic transient analysis and the Complex Eigenvalue Analysis (CEA). It is noteworthy that some researchers [9-11] use these two approaches to predict disc brake squeal. It was found that the CEA may lead to an over or under-estimation of the unstable modes, and that only the dynamic transient analysis allows to find the squeal behavior. However, because of the high computational cost of temporal methods, the CEA is then widely privileged in an industrial context. This method consists in the determination of the complex eigenvalues of the system around its nonlinear equilibrium as they characterize the possible appearance of an initial growing oscillatory behavior. These dynamic instabilities that may create unwanted squeal noise are so predicted.

The determination of the mechanism that induces the unstable vibrations is one of the most important step in studying brake systems and squeal. It is now admitted that there is no unique mathematical model in order to explain the mechanisms and dynamic phenomena of friction and instability in brake systems. According to some researchers [12-14], there are four general mechanisms for friction-induced system instability in brake 
systems: stick-slip, variable dynamic friction coefficient, sprag-slip [15] and coupling mechanism. The first two approaches rely on changes in the friction coefficient with relative sliding speed affecting the system stability. The last two approaches used kinematic constraints and modal coupling in order to develop the instability. In the present study the mode coupling phenomenon, that is caused by friction between the pads and the disc during braking, is privileged. In this case, instability can occur with a constant brake friction coefficient. We recall that even if many FEM studies used a constant value of this coefficient, some others offer sometimes further analysis on the influence of this friction coefficient by considering various effects such as a non-uniform contact pressure between the brake linings and the disc, the dependence of the friction coefficient versus velocity or temperature or the pads wear. The reader may refer to documents $[12-14,16-18]$ for details on these problematic that are not covered in this paper.

In recent years, the uncertainty analysis of brake squeal has become a hot topic. Indeed, some researchers demonstrate that considering uncertainty improves squeal prediction. For example, Tison et al. [2] statistically modelled the surfaces of the linings and studied the impact on stability. They showed that a modification of the surface of the linings has a high impact on the stability and can be considered as a key point for a more robust squeal prediction via CEA. Moreover, different methodologies have also be proposed to predict the squeal instability of industrial brake systems with uncertainties. Sarrouy et al. [19] used a multi-element polynomial chaos expansion coupled with a CEA analysis for the prediction of brake squeal with uncertainties. Nobari et al. [3] used kriging in order to predict the evolution of the real part of an unstable eigenvalue, and so the squeal propensity, assuming several uncertain parameters. On the same way, Nechak et al. [20] used kriging to realize a sensitivity analysis of the stability of a brake system. Lu et al. [21] used an interval analysis to model uncertainty on geometrical dimensions, material and loading properties of the brake system on a RSM build to surrogate the FEM. Then, they realized an optimization to identify a robust design. In 2017 [5], they used fuzzy logic combined to interval analysis in order to quantify the uncertainty in a FEM of a brake system. In 2017, Do et al. [22] used the fuzzy logic to manage the frictional contact problem in a numerical strategy to perform more rapidly multiple CEA and so the prediction of brake squeal.

Even if the determination of the assembled state of the brake system that secure the brake pads in the assembly is sometimes estimated in the first analysis step, the ability to use a representative model that takes into account the entire possible contact states between different components of the brake is only rarely implemented. In the study of the squeal noise phenomenon, it is considered that the frictional contact between the pads and the disc is the parameter of primal importance. However numerous components of the brake are in interaction with each other by various contact interfaces. If these contacts can be considered as secondary, no rigorous methodology has been proposed yet to study their impact on friction-induced squeal of a complete automotive disc brake model and the consequences of these contact states on the squeal propensity is quite poorly known. For this reason, a better understanding of considering internal contacts is of prior importance for the industrial and scientific community working on the automotive brake squeal.

It is with this mind that the present study proposes to inspect the influence of these internal contacts on the squeal propensity of an automotive brake and a strategy based on the genetic algorithm for the determination of the most influential contacts on brake squeal.

The paper is organized as follow. In a first part, the motivation of the present paper is developed. The automotive brake system under study is presented with its several interfaces that are frictional or not. The classical CEA is then briefly described. Once the problem is presented, preliminary results are given in a second part. This makes it possible to point out the influence of the friction coefficient at the interface between the pads and the disc, and also the influence of other potential internal frictional interfaces. To stay in an affordable computational cost, it is also shown that it is possible to consider only two different states, frictional or not, for each contact. Then, a deeper understanding of the role of internal contact interfaces on the system's stability is proposed in a third part by undertaking, first, a comparison of the unstable modes, and secondly, an analyze of the contact conditions associated to an instability in a given frequency range. In order to rank the effects of all the independent contact states, the restriction on the concept of the most unstable mode per frequency range is introduced. Finally, a strategy based on a Genetic Algorithm (GA) is adopted to define with a reduced computational cost the most unfavorable case (depending on the different internal contact states) to be considered in a design study concerning the squeal propensity. Motivation of such numerical strategy for an industrial point of view, as well as the advantages and limitations of the proposed methodology are discussed.

\section{Automotive brake system and stability analysis}

In this section, the Finite Element Model (FEM) of the automotive brake system under study will be first presented. Then brief reminders about the stability analysis are given. 


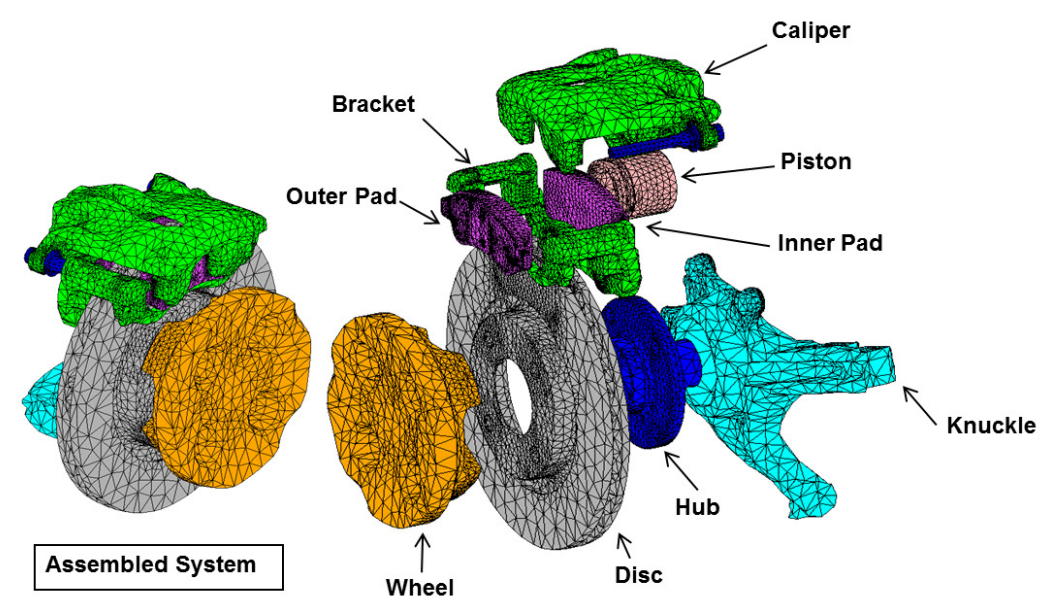

Figure 1: Finite element model developed with Abaqus - Assembled view (left) and exploded view (right)

Table 1: Material properties

\begin{tabular}{lllll}
\hline Element & Material & Young Modulus (GPa) & Poisson's coefficient & Density $\left(\mathrm{kg} / \mathbf{m}^{3}\right)$ \\
\hline Disc & Grey cast iron & 113 & 0.275 & 7075 \\
Caliper & Ductile cast iron & 175 & 0.275 & 7200 \\
Bracket & Ductile cast iron & 175 & 0.275 & 7200 \\
Pad & Steel & 210 & 0.29 & 7800 \\
\hline
\end{tabular}

\subsection{FEM brake system and motivation of the proposed study}

The brake system under study is shown in Figure 1. The hydraulically actuated disc brake consists of several components such as the disc, floating caliper, outer and inner pads, hub knuckle, piston and wheel. The braking action can be summarized as follows: during the braking process, hydraulic pressure is applied to the piston, and the inner pad is pushed by the piston until it contacts the disc. Then, the reaction forces push the caliper and the outer pad against the opposite side of the disc. The caliper that holds the two pads can move in parallel to the rotation axis of the disc when pressurizing and braking. This action create friction between the pads and disc. Sometimes squeal occurs when the brakes are applied. Even if it should not negatively affect brake stopping performance, instability and mode coupling due to friction may generate vibration of the brake components, especially the pads and disc.

Traditionally the friction coefficient at the pad/disc interface is considered as one of the most influential parameter on the automotive brake system stability. Thereby, the majority of numerical simulations for the prediction of squeal propensity are performed by considering only the two friction surfaces between the pads and disc for different values of this friction coefficient and neglecting other friction surfaces at other components of the automotive brake system.

However, many contact interfaces are present in an automotive brake system. During braking, different components can interact with each other through different contact interfaces. It is particularly true for the pads that can be in contact with three components in addition to the disc, namely the piston, the caliper and the bracket. The status of each contact is directly related to the position of the pads in the automotive brake system, which are supposed to be free of movement. It can be noted that a new static equilibrium of the brake system may appear if a state of contact is modified (in particular if the contact is considered as frictional or not). This leads to a new potential state of the brake system with respect to its stability.

The implementation and the validation of the FEM is not the main concern of this study. For more details, please see $[1,23]$. The materials of the different components of the brake and their properties used in the FEM are given in Table 1. A linear penalty method is used for the contact constraint enforcement and the classic Coulomb's law is used for all the frictional interfaces. The braking pressure and the disc rotation velocity are constant. The hydraulic system is represented by an effort applied on the back of the piston and on the caliper surface. Considering more specifically the boundary conditions, the brake system is connected to the car's frame by considering a zero displacement condition imposed at the connection of the knuckle with the frame. The connection between the knuckle and the tie rod is modelled by ball joints. The rotation between the hub and the knuckle is stacked. We will only explain the inclusion of various contact states in the 

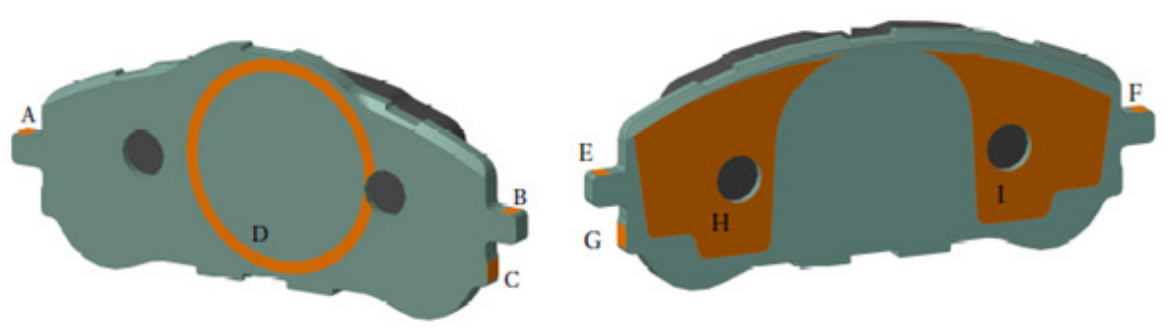

Figure 2: Contact Interfaces of internal (left) and external (right) pads

Table 2: Contact parameters

\begin{tabular}{clc}
\hline Interface letter & Type of contact & Parameter \\
\hline A & Inner Top Radial Contact & RHI \\
B & Inner Bottom Radial Contact & RBI \\
C & Inner Bracket-Pad Contact & RCBI \\
D & Pad-Piston & PP \\
E & Outer Bottom Radial Contact & RBE \\
F & Outer Top Radial Contact & RHE \\
G & Outer Bracket-Pad Contact & RCBE \\
H & Bottom Caliper-Pad Contact & DB \\
I & Top Caliper-Pad Contact & DH \\
\hline
\end{tabular}

following. Considering the FEM under study, the three contact interactions taken into account (respectively named piston/pad, bracket/pad and caliper/pad) lead us to consider nine new possible contact interfaces, as indicated in Figure 2 (see the letters A, B, C, D E, F G, H and I) and summarized in Table 2.

Considering these three contact interactions between the piston and the pad, the bracket and the pad and the caliper and the pad, the non-linear dynamic problem of the brake system can be written as:

$$
\mathbf{M} \ddot{\mathbf{X}}+\mathbf{C} \dot{\mathbf{X}}+\mathbf{K X}+\mathbf{F}_{\text {piston } / \text { pad }}(\mathbf{X})+\mathbf{F}_{\text {bracket } / \text { pad }}(\mathbf{X})+\mathbf{F}_{\text {caliper } / \text { pad }}(\mathbf{X})+\mathbf{F}_{\text {nl }}(\mathbf{X})=\mathbf{F}_{\text {ext }}
$$

where $\mathbf{X}$ is the generalized displacement vector, and the dot represents the derivative with respect to time. $\mathbf{M}, \mathbf{C}$ and $\mathbf{K}$ are mass, damping and structural stiffness matrices, respectively. $\mathbf{F}_{\text {ext }}$ represents the external efforts, here it corresponds to the pressure applied on the piston and the caliper. $\mathbf{F}_{\text {piston/pad }}(\mathbf{X}), \mathbf{F}_{\text {bracket } / \mathbf{p a d}}(\mathbf{X})$ and $\mathbf{F}_{\text {caliper/pad }}(\mathbf{X})$ define the equivalent stiffness contributions for the interfaces of the two sub-systems under consideration (see $[1,23]$ for more details). In the case of contact for a given interface, normal forces and friction forces are generated. The detachment (i.e. switching from a contact state to a non-contact state at the selected interfaces) is allowed for each interface. $\mathbf{F}_{\mathbf{n}}$ are the non-linear efforts and contains contributions from both the non-linear contact forces and frictional forces at the pad/disc contact interfaces (see [23] for more details). Each contact interface is characterized by a classical Coulomb's law with a constant friction coefficient.

So the motivation and the goal of the present paper is to study the impact of considering all of these contact surfaces on the automotive brake system stability.

\subsection{Stability analysis}

As previously mentioned in the introduction, the CEA is the classical and privileged method for the squeal prediction in car industry. It is based on the analysis of the eigenvalues of the system linearized around its static equilibrium. The presence of the friction forces may introduce non-symmetric terms in the system's matrices, which can lead to instabilities. The full CEA procedure is detailed bellow.

By defining the global stiffness matrix $\mathbf{K}_{\mathbf{n l}, \mathbf{U}_{\mathbf{S}}}$ due to not only the structural components of the brake system but also the three contact interfaces as follow:

$$
\mathbf{K}_{\text {nl, } \mathbf{U}_{\mathrm{S}}} \mathbf{U}_{\mathbf{S}}=\mathbf{K U}_{\mathbf{S}}+\mathbf{F}_{\text {piston } / \text { pad }}\left(\mathbf{U}_{\mathbf{S}}\right)+\mathbf{F}_{\text {bracket } / \text { pad }}\left(\mathbf{U}_{\mathbf{S}}\right)+\mathbf{F}_{\text {caliper } / \text { pad }}\left(\mathbf{U}_{\mathbf{S}}\right)
$$

where $\mathbf{K}_{\mathbf{n l}, \mathbf{U}_{\mathbf{S}}}$ corresponds to the linearized stiffness matrix at the vicinity of the non-linear equilibrium point. This contribution includes the possibility of contact or loss of contact for the three internal contact interfaces 
between the pad and the piston, the bracket and the pad, and the caliper and the pad. The determination of the non-linear static equilibrium $\mathbf{U}_{\mathbf{S}}$ is then given by:

$$
\mathbf{K}_{\mathrm{nl}, \mathbf{U}_{\mathrm{S}}} \mathbf{U}_{\mathrm{S}}+\mathbf{F}_{\mathrm{nl}}\left(\mathbf{U}_{\mathrm{S}}\right)=\mathbf{F}_{\text {ext }}
$$

The system is studied around the equilibrium point $\mathbf{U}_{\mathbf{S}}$ by assuming a small perturbation $\overline{\mathbf{X}}$, so that $\mathbf{X}=\mathbf{U}_{\mathbf{S}}+\overline{\mathbf{X}}$. Then, the non-linear force $\mathbf{F}_{\mathbf{n l}}$ can be linearised to the first order around the equilibrium point $\mathbf{U}_{\mathbf{S}}$. By denoting $\mathbf{J}_{\mathbf{n l}}$ the Jacobian matrix of $\mathbf{F}_{\mathbf{n l}}$ at the point $\mathbf{U}_{\mathbf{S}}$, it comes: $\mathbf{F}_{\mathbf{n l}}(\mathbf{X})=\mathbf{F}_{\mathbf{n l}}\left(\mathbf{U}_{\mathbf{S}}\right)+\mathbf{J}_{\mathbf{n l}} \overline{\mathbf{X}}$. The previous relation of Equation 1 can be rewritten in the following form:

$$
\mathbf{M} \ddot{\overline{\mathbf{X}}}+\mathbf{C} \dot{\overline{\mathbf{X}}}+\left(\mathbf{K}_{\mathbf{n l}, \mathbf{U}_{\mathrm{S}}}+\mathbf{J}_{\mathbf{n l}}\right) \overline{\mathbf{X}}=\mathbf{0}
$$

The eigenvalue problem to be solved is then given by eigenvalue problem:

$$
\left(\lambda^{2} \mathbf{M}+\lambda \mathbf{C}+\left(\mathbf{K}_{\mathbf{n l}, \mathbf{U}_{\mathbf{S}}}+\mathbf{J}_{\mathbf{n l}}\right)\right) \mathbf{\Psi}=\mathbf{0}
$$

Due to friction, the matrix $\mathbf{J}_{\mathbf{n l}}$ is non-symmetric. So running CEA produces complex eigenvalues $\lambda_{j}=$ $a_{j}+i \omega_{j}$ and complex eigenvectors $\boldsymbol{\Psi}_{j} . \omega_{j}$ defines the pulsation of the mode $\boldsymbol{\Psi}_{j}$ and $a_{j}$ the associated real part. The system is considered as unstable if at least one eigenvalue has a positive real part. If all the real parts are negative the equilibrium point remains stable. Hence, the squeal propensity of a brake system reflects the tendency of the brake to squeal. Based on the CEA, it corresponds to the ratio between the number of unstable configurations (eigenvalues with positive real parts) and the number of samples. The higher is the positive real part, the higher is the initial growth rate of the dynamic response when the static sliding equilibrium is perturbed. We recall that the CEA analysis may lead to an underestimation or an over-estimation of the unstable modes observed during squeal noise because the linearized stability around an initial equilibrium point with a defined contact state is not valid during friction-induced vibration and noise. So the nonlinear transient self-excited vibrations can become very complex and include more or less unstable modes due to the nonlinear contact and loss of contact interactions at the frictional interface. Therefore, a complete nonlinear simulation should be performed in addition to the stability analysis to estimate the real most unstable configuration in regard to squeal noise [24-27]. This is out of reach of the present study because of the numerical implementation difficulty and the prohibitive calculation times associated with the estimation of friction-induced vibration and noise for a FEM brake system.

\section{Preliminary results and conclusions on the influence of the friction coefficient at internal contact interfaces}

A classical methodology to study the squeal propensity of an automotive brake system consists in running several CEA for different values of the friction coefficient. In order to focus only on the influence of the different interfaces, the damping is neglected in the present paper. Indeed, the role of damping remains an extremely important issue in the squeal problem and several studies on the effect of damping on stability and on self-excited vibrations have been proposed. We recall that damping proves to have two different effects: a "lowering effect" that tends to stabilize the system and a "smoothing effect" that may induce a destabilizing effect regarded for a long time as a "paradox". However, considering that investigating the effect of damping is out of the scope of the present study, the reader can refer to the following paper [28-30]. Therefore, in order to have a first trend on the influence of the friction coefficient (denoted $\mu_{\text {intern }}$ ) at the nine internal contact interfaces, the following strategy is first proposed: all the considered internal contacts presented in Table 2 are characterized by the same friction coefficient $\mu_{\text {intern }}$. Two specific cases are undertaken:

- Case 1: a variation of the friction coefficient $\mu_{\text {intern }}$ is considered at the nine internal contact interfaces (from 0 to 1 by keeping the same value for each internal interface) for three different values of $\mu_{\text {pad-disc }}$ : $\mu_{\text {pad-disc }}=0.3, \mu_{\text {pad-disc }}=0.5$ or $\mu_{\text {pad-disc }}=0.7$;

- Case 2: four specific configurations versus $\mu_{\text {intern }}$ are investigated with a variation of the friction coefficient $\mu_{\text {pad-disc }}$ from 0 to 1 . The four different values of the friction coefficient $\mu_{\text {intern }}$ at the nine internal contact interfaces are $\mu_{\text {intern }}=0, \mu_{\text {intern }}=0.15, \mu_{\text {intern }}=0.3$ and $\mu_{\text {intern }}=0.5$. It is reminded that the nine internal interfaces have the same value of $\mu_{\text {intern }}$ for each configuration.

The evolution of the eigenvalues in the complex plan are displayed in Figure 3 for Case 1 . The different mode shapes associated to the different instabilities are very similar when $\mu_{\text {intern }}$ varies, and the location of the unstable large-amplitude vibrations are constant. For the sake of brevity, they are not represented here. 


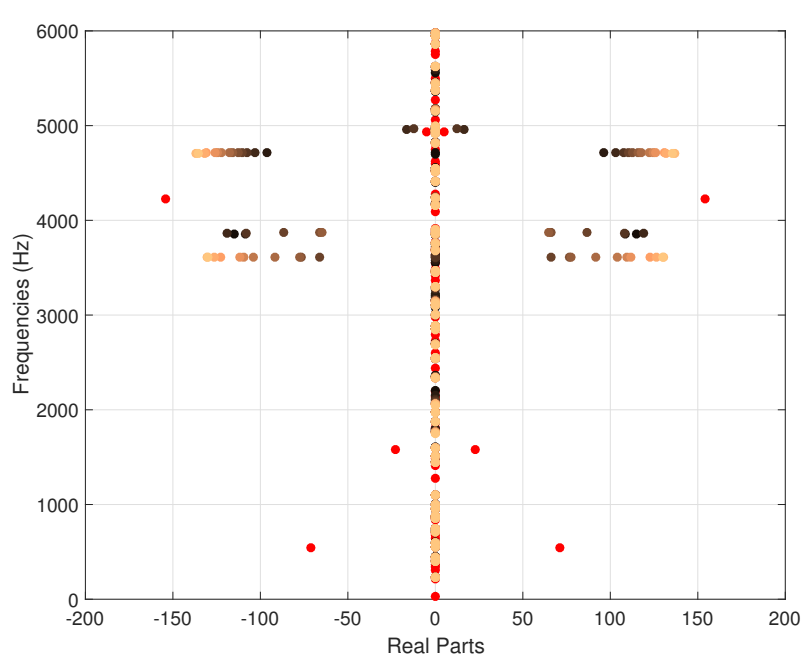

(a)

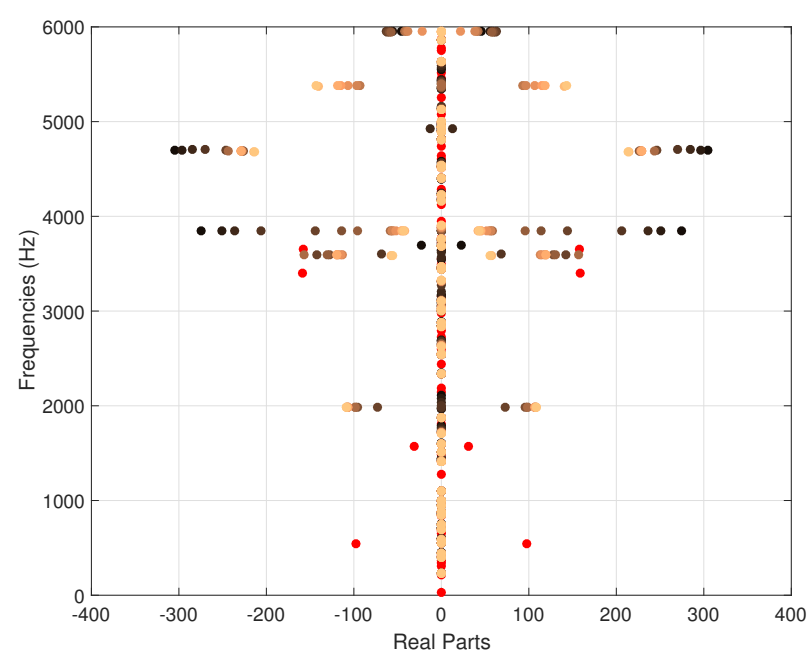

(b)

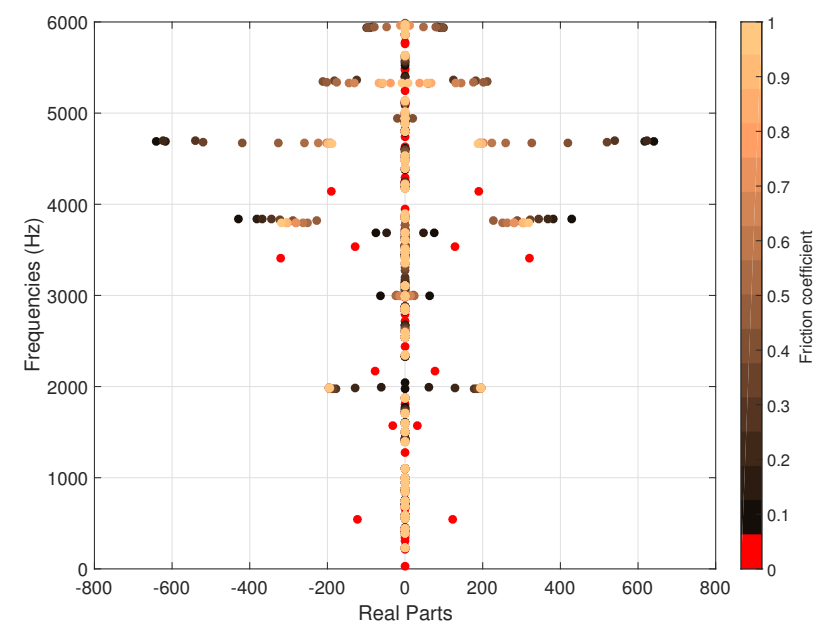

(c)

Figure 3: Evolution of complex eigenvalues in the complex plan assuming a variation of the friction coefficient $\mu_{\text {intern }}$ at the interfaces for three different values of $\mu_{\text {pad-disc }}$ : (a) $\mu_{\text {pad-disc }}=0.3$, (b) $\mu_{\text {pad-disc }}=0.5$, (c) $\mu_{\text {pad-disc }}=0.7$

Similarly, Table 3 summarizes the number of instabilities, the range of friction coefficient values for which the mode is unstable (noted $\left[\boldsymbol{\mu}_{\mathbf{u}, \mathbf{i n f}} ; \boldsymbol{\mu}_{\mathbf{u}, \mathbf{s u p}}\right]$ ) and the associated frequency of the unstable mode for the four configurations tested in Case 2.

All these preliminary results of Cases 1 and 2 demonstrate the influence of the friction coefficient $\mu_{\text {intern }}$. Showing Figure 3, it is also observed that even if the variation of $\mu_{\text {intern }}$ does not drastically affect the frequency value of the unstable modes, a significant effect is visible on the variation of the real parts. For instance, if $\mu_{\text {pad-disc }}=0.5$, at $3900 \mathrm{~Hz}$, the real part varies from about 30 to about 290 . Of course as previously discussed, Figure 3 illustrates the fact that the friction coefficient $\mu_{\text {intern }}$ impacts the number of unstable modes. Hence, when $\mu_{\text {pad-disc }}=0.5$, at about $5300 \mathrm{~Hz}$, the mode is unstable only when $\mu_{\text {intern }}$ is superior to 0.6. Comparing cases where $\mu_{\text {intern }} \neq 0$, the main difference is observed on the real part of the instabilities and on the number of instabilities. Indeed when $\mu_{\text {intern }}$ increases (but is non zero), the number of unstable modes increases. As summarized in Table 3, when $\mu_{\text {intern }}=0.15$ only four instabilities are present, whereas six instabilities (seven respectively) exist when $\mu_{\text {intern }}=0.3\left(\mu_{\text {intern }}=0.5\right.$ respectively). Moreover, a variation of $\mu_{\text {intern }}$ implies a significant variation of the real parts of unstable modes. Indeed, considering the instability around $[4700-4800] \mathrm{Hz}$, the maximum of the real part may decrease or increase when $\mu_{\text {intern }}$ increases according to the value of $\mu_{\text {pad-disc }}$. Hence, when $\mu_{\text {pad-disc }}=0.3$, the increasing of $\mu_{\text {intern }}$ has a destabilizing effect, when it has a stabilizing effect for $\mu_{\text {pad-disc }}=0.5$ and $\mu_{\text {pad-disc }}=0.7$. (see and compare Figures 3 for example).

It is also observed in Table 3 that the value of the critical point and the frequency of each unstable mode are very dependent on both the values of $\mu_{\text {intern }}$ and $\mu_{\text {pad-disc }}$. So the effect of $\mu_{\text {intern }}$ can not be considered 
Table 3: Instabilities for different internal friction coefficients

\begin{tabular}{|c|c|c|c|c|}
\hline & $\begin{array}{l}\text { Instability } \\
\text { number }\end{array}$ & Frequency $(\mathbf{H z})$ & $\begin{array}{c}\text { Maximum Real } \\
\text { Part }\end{array}$ & {$\left[\mu_{\mathrm{u}, \mathrm{inf}} ; \mu_{\mathrm{u}, \mathrm{sup}}\right]$} \\
\hline \multirow{13}{*}{$\mu_{\text {intern }}=0$} & 1 & 540.3 & 133.3 & {$[0.1 ; 1]$} \\
\hline & 2 & 676.7 & 62.92 & {$[0.9 ; 1]$} \\
\hline & 3 & 1574 & 33.14 & {$[0.2 ; 1]$} \\
\hline & 4 & 2172 & 216.9 & {$[0.75 ; 1]$} \\
\hline & 5 & 3431 & 842.8 & {$[0.45 ; 1]$} \\
\hline & 6 & 3461 & 350.1 & {$[0.7 ; 1]$} \\
\hline & 7 & 3662 & 181.3 & {$[0.35 ; 0.55]$} \\
\hline & 8 & 4168 & 624.7 & {$[0.7 ; 1]$} \\
\hline & 9 & 4239 & 236.5 & {$[0.25 ; 0.45]$} \\
\hline & 10 & 4242 & 168.8 & {$[0.8 ; 0.95]$} \\
\hline & 11 & 4598 & 72.81 & {$[0.9 ; 1]$} \\
\hline & 12 & 4742 & 141.6 & {$[1 ; 1]$} \\
\hline & 13 & 5355 & 374.9 & {$[0.7 ; 1]$} \\
\hline \multirow{5}{*}{$\mu_{\text {intern }}=0.15$} & 1 & 3119 & 92.08 & {$[1 ; 1]$} \\
\hline & 2 & 3446 & 34.52 & {$[1 ; 1]$} \\
\hline & 3 & 3633 & 16.06 & {$[0.6 ; 0.6]$} \\
\hline & 4 & 3810 & 192.8 & {$[0.35 ; 1]$} \\
\hline & 5 & 5389 & 658.2 & {$[0.4 ; 1]$} \\
\hline \multirow{6}{*}{$\mu_{\text {intern }}=0.3$} & 1 & 803.4 & 40.52 & {$[0.65 ; 1]$} \\
\hline & 2 & 3106 & 100.9 & {$[0.65 ; 1]$} \\
\hline & 3 & 3443 & 48.27 & {$[1 ; 1]$} \\
\hline & 4 & 3669 & 30.38 & {$[1 ; 1]$} \\
\hline & 5 & 3816 & 178 & {$[0.35 ; 1]$} \\
\hline & 6 & 5365 & 627.6 & {$[0.65 ; 1]$} \\
\hline \multirow{8}{*}{$\mu_{\text {intern }}=0.5$} & 1 & 802.9 & 72.43 & {$[0.65 ; 1]$} \\
\hline & 2 & 3002 & 32.95 & {$[0.8 ; 1]$} \\
\hline & 3 & 3093 & 82.75 & {$[0.6 ; 1]$} \\
\hline & 4 & 3681 & 227.5 & {$[0.75 ; 1]$} \\
\hline & 5 & 3824 & 145.3 & {$[0.4 ; 1]$} \\
\hline & 6 & 4331 & 104.3 & {$[0.65 ; 1]$} \\
\hline & 7 & 5241 & 133.9 & {$[0.85 ; 0.95]$} \\
\hline & 8 & 5324 & 302.7 & {$[1 ; 1]$} \\
\hline
\end{tabular}

as negligible. It is not intuitive and it can be complex to analyze. However, by analyzing all the results, a first trend emerges.

However, a significant trend is emerging: the system's behavior is very different if $\mu_{\text {intern }}=0$ or $\mu_{\text {intern }} \neq 0$. In other words, two cases are easily differentiated, the first corresponding to the case where the coefficient of friction $\mu_{\text {intern }}$ is not zero, the second where it is zero, which corresponds to contact or non-contact conditions. In the following, the terminology "non-frictional state" will be used to describe the case for $\mu_{\text {intern }}=0$. The terminology "frictional state" will be used to describe the case for $\mu_{\text {intern }} \neq 0$.

Now a first intermediate conclusion can be made: this preliminary study demonstrates the influential role of internal contacts of a brake system on its stability. It is obvious that in a process of characterizing the squeal propensity of a brake system, they cannot be neglected. In view of the numerical cost that so many additional parameters may imply, this conclusion can be a tedious one and a strategy to take these contacts into account at a reasonable cost is necessary. As previously discussed, the first strong trend lies on the fact that the system can be studied with two different states for each internal contact, namely frictional or not. In this preliminary study we recall that we considered a simplified case for which all internal contact interfaces behave in the same way. In a more general context where each internal contact can have a specific configuration (no friction with $\mu_{\text {intern }}=0$ or friction with $\mu_{\text {intern }} \neq 0$ ), a number of $2^{9}=512$ different configurations (i.e. nine internal

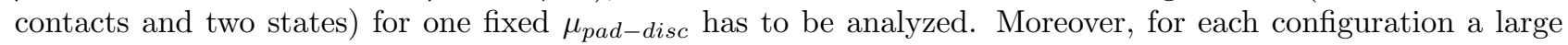
number of CEA is required for the prediction of the $\mu_{p a d-d i s c}$ induced instabilities of the industrial FEM. 


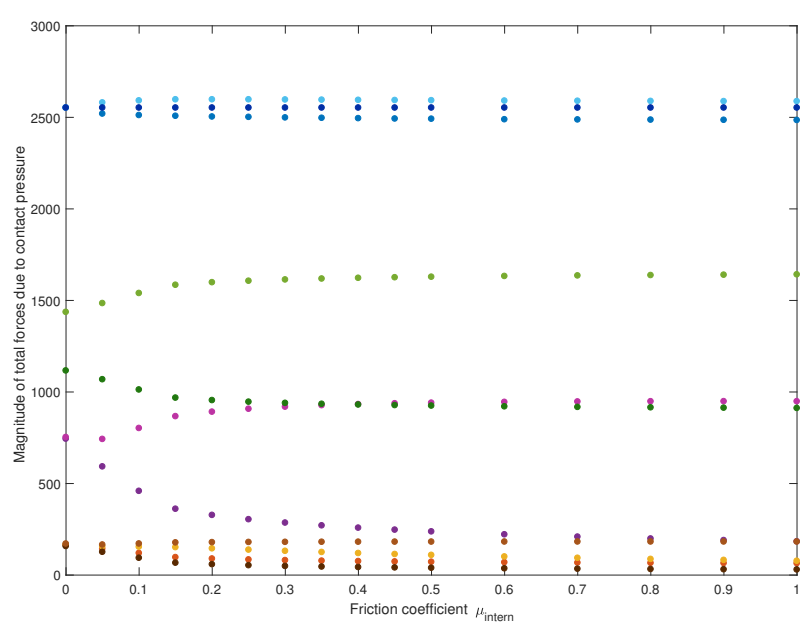

(a)

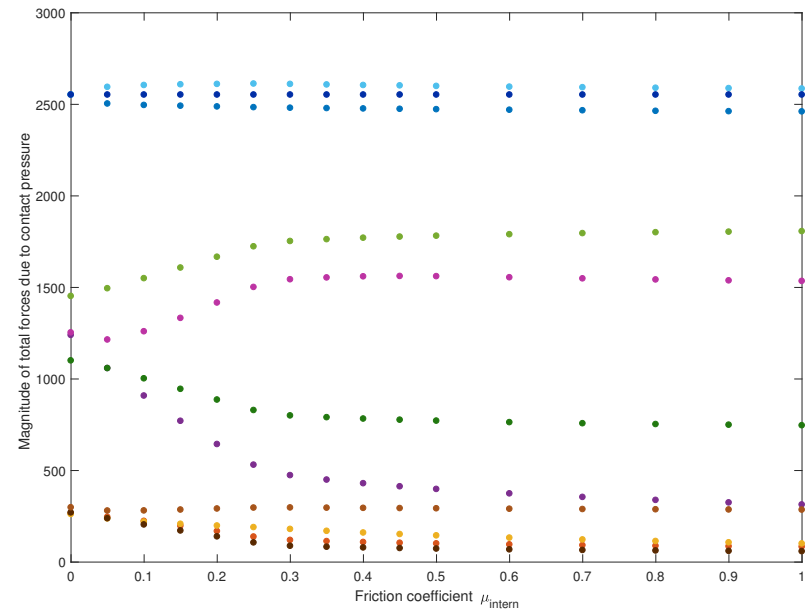

(b)

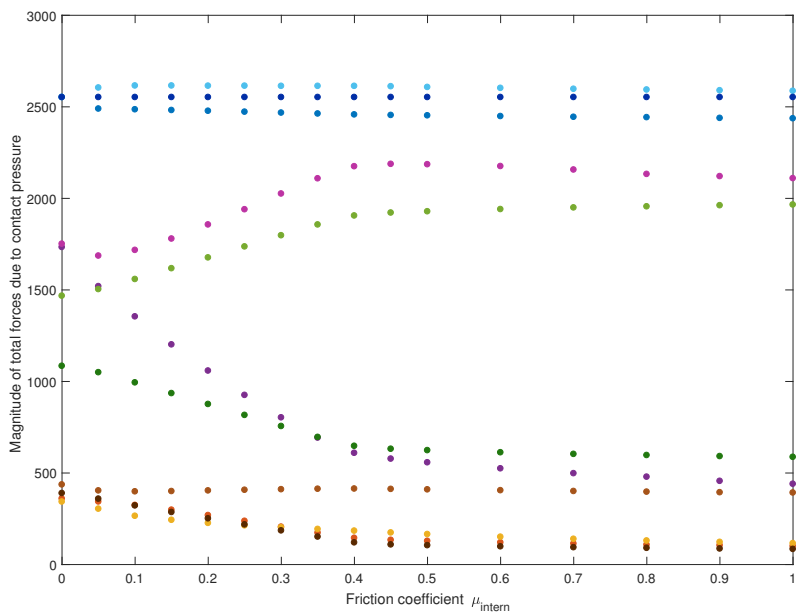

(c)

Figure 4: Evolution of the magnitude of the total force due to contact pressure at the different interfaces versus the friction coefficient $\mu_{\text {intern }}$ at the interfaces for three different values of $\mu_{\text {pad-disc }}$ : (a) $\mu_{\text {pad-disc }}=0.3$, (b) $\mu_{\text {pad-disc }}=0.5$, (c) $\mu_{\text {pad-disc }}=0.7$ - Contacts: $\bullet \mathrm{RCBE}, \bullet \mathrm{RCBI}, \bullet \mathrm{DB}, \bullet \mathrm{DH}, \bullet \mathrm{MU}$ ext, $\bullet \mathrm{MU}$ int, $\bullet \mathrm{PP}, \bullet$ $\mathrm{RBE}, \bullet \mathrm{RBI}, \bullet \mathrm{RHE}, \bullet \mathrm{RHI}$

At this stage of the study it is essential to remember that the configuration $\mu_{\text {intern }} \neq 0$ can be performed by using different friction values $\mu_{\text {intern }}$. This choice can then impact the stability results. Also in order to carry out a more detailed study on the independent effect of each internal contact interface, we propose to quickly explain the choice that has been made on the value of $\mu_{\text {intern }}$ for the rest of the study. In order to argue our choice, the evolution of the magnitude of the total force due to contact pressure at the different internal interfaces are displayed on Figure 4. It is observed that the magnitude of the total force in each contact changes and reaches a steady state above a certain threshold. An increasing of $\mu_{p a d-d i s c}$ raises this threshold. For some contacts, the variation between the initial state at $\mu_{\text {intern }}=0$ and the steady state is weak, as for PP or MU int/ext. But for others, the variation can be large and becomes larger when $\mu_{\text {pad-disc }}$ increases, as for RCBE which has an initial total magnitude of pressure forces equals to $1550 \mathrm{~N}$ when $\mu_{\text {intern }}=0$ and about $500 \mathrm{~N}$ when $\mu_{\text {intern }}=1$, if $\mu_{\text {pad-disc }}=0.7$. In light of these results, to choose the value of the non-zero friction coefficient $\mu_{\text {intern }}$, two different choices are possible. The first one is a friction coefficient $\mu_{\text {intern }}$ on which the system has reached its steady state. However the steady state's threshold varies with $\mu_{\text {pad-disc }}$ and so it could be difficult to determine a value for which the system is always at its steady state, not to mention that this threshold value is initially unknown and may depend of the contact. The second choice is to take a value of the friction coefficient $\mu_{\text {intern }}$ for which the force magnitudes due to contact pressure at the different interfaces are in a transitional state on regard to the friction coefficient $\mu_{\text {intern }}$. It is this second choice that we retained for the rest of the study. The value of the friction coefficient $\mu_{\text {intern }}$ is taken equal to 0.15. Even if this choice appears to be close to the threshold value if $\mu_{\text {pad-disc }}=0.3$, the design of automotive brake systems are generally performed 


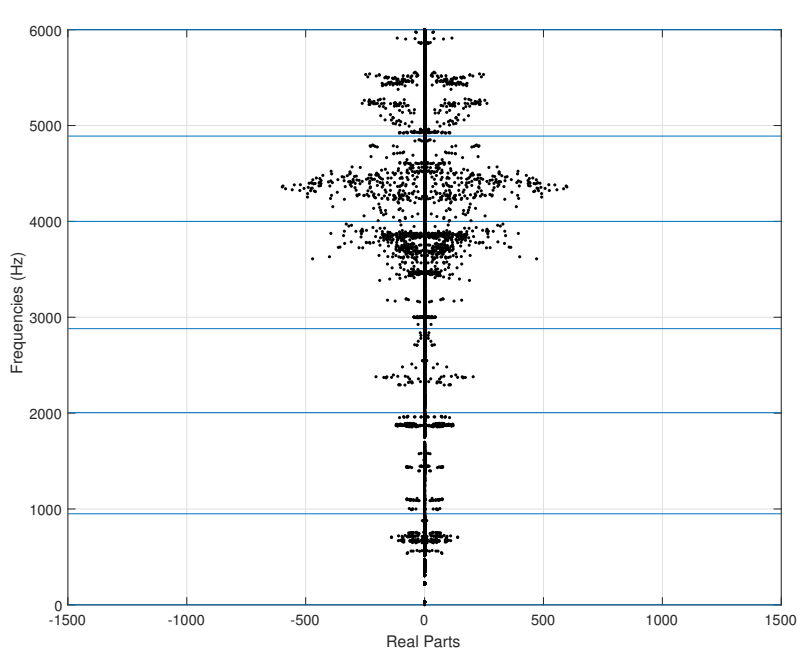

(a)

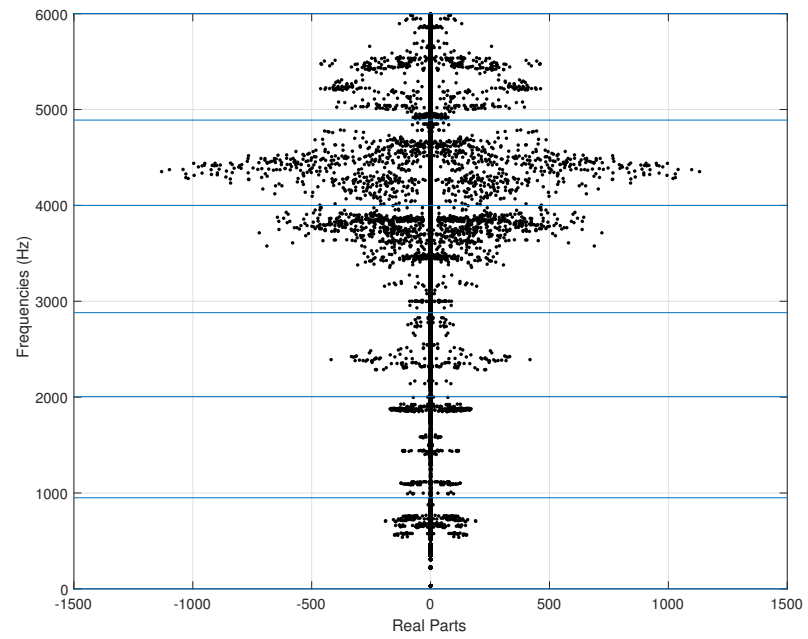

(b)

Figure 5: Eigenvalues in the complex plan and frequency ranges for $\mu_{\text {pad } / \text { disc }}=0.3$ (left) and $\mu_{\text {pad } / \text { disc }}=0.5$ (right)

for values of the friction coefficient $\mu_{\text {pad-disc }}$ superior to 0.3 . Moreover, by taking $\mu_{\text {intern }}=0.15$, we notice on Figure 3 that the majority of instabilities have already emerged. As previously observed, the real part of eigenvalues varies with $\mu_{\text {intern }}$ so this cannot be a criterion to choose its value. The frequencies are, on the other hand, not very sensitive to this value. In a nutshell, by taking $\mu_{\text {intern }}=0.15$, the unstable modes and their frequencies are identified which is consistent in relation to our motivation and the purpose of our study.

\section{On a better understanding of the role of internal contact interfaces}

\subsection{Motivation}

Based on the above results and to stay in affordable computational times, the choice can be made to limit the study to the consideration of two contact states for each of the nine interfaces: a non-friction state with a zero friction coefficient $\mu_{\text {intern }}$, and a friction state with a friction coefficient $\mu_{\text {intern }}$ equals to 0.15 . If all combinations of internal contacts are considered for a fixed $\mu_{\text {pad-disc }}$, significant differences on the associated squeal propensities can nevertheless be observed. This is illustrated in Figures 5 where the friction coefficient $\mu_{\text {pad-disc }}$ is constant and fixed at 0.3 for Figure 5(a) and at 0.5 for Figure 5(b). The 512 associated CEA configurations are performed for both cases and the obtained eigenvalues are superimposed and displayed in the complex plan. The influence of the contact conditions appears to be an important factor in the prediction of squeal frequencies. For both cases, some groups of eigenvalues can be made: for example between $[1080 ; 1120] \mathrm{Hz}$ or $[1850 ; 2000] \mathrm{Hz}$. But for frequencies higher than $3300 \mathrm{~Hz}$, a strong dispersion is observed. It makes the tracking of the unstable modes versus the configurations a very hard task. A first conclusion is the high sensitivity of the unstable eigenvalues to contact states. So it is necessary to investigate more precisely those results by analyzing the impact of each of the nine internal contact interfaces on the generation or not of unstable modes. The effects of the frictional behavior between the other parts on the motion of the disc-pad interface is not presented in this study since they are outside the scope of the study. The main objective of the present study is to undertake a global vision of the effect of contact interfaces and the determination of the most influential contacts between the different components of an automotive brake system.

In order to conduct such an analysis, this section is organized as follow: in a first time, the shapes of unstable modes are studied and compared in regard to different internal contacts. In a second time, the contact conditions associated to an unstable frequency are analyzed. The main objective is to identify recurrent unstable modes, or to identify contacts that are systematically involved in some instabilities.

\subsection{Analysis of the unstable modes versus internal contacts}

The first step consists in the comparison of the unstable modes. For this purpose, a group of 25 representative configurations is retained. Their characteristics are given in Table 4 and the unstable frequencies are given in Table 5. For information, the first configuration corresponds to the case where all internal contacts are not 
Table 4: Characteristics of the 25 configurations under study

\begin{tabular}{cclllllllll}
\hline \multirow{2}{*}{ Configuration } & $\begin{array}{c}\text { Number of } \\
\text { instabilities }\end{array}$ & RHI & RHE & RCBE & RCBI & RBE & RBI & PP & DB & DH \\
\hline CFG1 & 11 & 0 & 0 & 0 & 0 & 0 & 0 & 0 & 0 & 0 \\
CFG2 & 7 & 0.15 & 0.15 & 0.15 & 0.15 & 0.15 & 0.15 & 0.15 & 0.15 & 0.15 \\
CFG3 & 5 & 0.15 & 0.15 & 0.15 & 0 & 0.15 & 0 & 0.15 & 0.15 & 0.15 \\
CFG4 & 9 & 0 & 0.15 & 0.15 & 0 & 0.15 & 0.15 & 0.15 & 0 & 0 \\
CFG5 & 8 & 0 & 0.15 & 0.15 & 0 & 0.15 & 0.15 & 0 & 0 & 0 \\
CFG6 & 6 & 0 & 0.15 & 0.15 & 0 & 0.15 & 0 & 0 & 0.15 & 0.15 \\
CFG7 & 6 & 0.15 & 0 & 0 & 0.15 & 0.15 & 0.15 & 0.15 & 0.15 & 0.15 \\
CFG8 & 6 & 0 & 0 & 0.15 & 0.15 & 0.15 & 0.15 & 0.15 & 0.15 & 0.15 \\
CFG9 & 7 & 0.15 & 0.15 & 0 & 0 & 0 & 0 & 0.15 & 0.15 & 0.15 \\
CFG10 & 7 & 0.15 & 0.15 & 0 & 0 & 0 & 0 & 0.15 & 0 & 0 \\
CFG11 & 6 & 0 & 0.15 & 0 & 0 & 0 & 0.15 & 0.15 & 0 & 0.15 \\
CFG12 & 6 & 0 & 0.15 & 0 & 0 & 0 & 0.15 & 0.15 & 0.15 & 0 \\
CFG13 & 9 & 0 & 0 & 0 & 0 & 0 & 0 & 0.15 & 0 & 0 \\
CFG14 & 5 & 0 & 0 & 0 & 0 & 0 & 0 & 0.15 & 0 & 0.15 \\
CFG15 & 5 & 0 & 0 & 0 & 0 & 0 & 0 & 0.15 & 0.15 & 0.15 \\
CFG16 & 6 & 0 & 0.15 & 0 & 0 & 0 & 0 & 0.15 & 0.15 & 0.15 \\
CFG17 & 6 & 0.15 & 0.15 & 0.15 & 0 & 0 & 0 & 0.15 & 0.15 & 0.15 \\
CFG18 & 8 & 0.15 & 0.15 & 0.15 & 0.15 & 0 & 0 & 0.15 & 0.15 & 0.15 \\
CFG19 & 7 & 0.15 & 0.15 & 0.15 & 0.15 & 0.15 & 0 & 0.15 & 0.15 & 0.15 \\
CFG20 & 6 & 0.15 & 0.15 & 0.15 & 0.15 & 0.15 & 0.15 & 0 & 0 & 0 \\
CFG21 & 6 & 0.15 & 0.15 & 0.15 & 0.15 & 0.15 & 0.15 & 0 & 0 & 0.15 \\
CFG22 & 6 & 0.15 & 0.15 & 0 & 0 & 0 & 0 & 0.15 & 0 & 0.15 \\
CFG23 & 4 & 0.15 & 0.15 & 0 & 0 & 0.15 & 0.15 & 0.15 & 0.15 & 0.15 \\
CFG24 & 6 & 0 & 0 & 0.15 & 0.15 & 0 & 0 & 0.15 & 0.15 & 0.15 \\
CFG25 & 6 & 0 & 0 & 0 & 0 & 0.15 & 0.15 & 0.15 & 0.15 & 0.15 \\
\hline
\end{tabular}

frictional and the second where all are frictional. The eleventh and twelfth configurations correspond to the two most unstable configurations (i.e. with the maximum real part). In order to compare the different unstable modes observed for each configuration, it is necessary to take a friction coefficient $\mu_{\text {pad-disc }}$ at the pad/disc interface that makes a maximum of instabilities emerge. As seen in Table 3, the higher the friction coefficient is, the higher the number of instability is. For this reason, the friction coefficient at the pad/disc interface $\mu_{\text {pad-disc }}$ is chosen equal to 0.9 in order to get as many instabilities as possible.

The different unstable modes observed on the twenty-five configurations tested are compared by applying a Modal Assurance Criterion (MAC). The MAC corresponds to a measure of the degree of linearity between two modes. A high value of the MAC indicates that the mode shapes are correlated, and so modes can be paired and it is possible to consider that they correspond to the same instability. A low value, indicates that mode shapes are orthogonal (i.e. uncorrelated), and so that they do not correspond to the same instability. The MAC value between two mode shapes $\boldsymbol{\Phi}_{1}$ and $\boldsymbol{\Phi}_{2}$ is given by:

$$
\operatorname{MAC}\left(\boldsymbol{\Phi}_{1}, \boldsymbol{\Phi}_{2}\right)=\left(\frac{\left|\boldsymbol{\Phi}_{1}^{*} \boldsymbol{\Phi}_{2}\right|}{\left\|\boldsymbol{\Phi}_{1}\right\|\left\|\boldsymbol{\Phi}_{2}\right\|}\right)^{2}
$$

where ${ }^{*}$ designate the conjugate transpose of a complex vector.

For the sake of simplicity, the unstable mode shapes of the twenty-five configurations tested are concatenated and an AutoMAC is performed. The AutoMAC matrix obtained is displayed Figure 6. The different configurations are separated by a red dot line. The block at the $i^{\text {th }}$ row and at the $j^{\text {th }}$ column corresponds to the MAC matrix between the unstable modes of configurations $i$ and $j$. The blocks on the diagonal are the AutoMAC of each configuration.

Except for a few exceptions, there is no correspondence between the different unstable modes of the different configurations. Some configurations seem to have similar unstable modes (for example configurations 2 and 19 or 25 and 8), but in most cases there is no correlation. The Frequency MAC (FMAC), which is a MAC with a frequency scale, is displayed Figure 7. It appears that mode shapes with a high correlation have close natural frequencies. Indeed, MAC values around 1 are at proximity of the diagonal. But it is also possible to find two modes with similar natural frequencies and totally different mode shapes. This study shows that the unstable modes are different for each configuration and are sensitive to the contact state at each of the nine internal interfaces. It means that it is actually not possible to track an unstable mode versus the different configurations. 
Table 5: Frequencies of unstable modes of the 25 configurations

\begin{tabular}{|c|c|c|c|c|c|c|c|c|c|c|c|}
\hline \multirow{2}{*}{ Configuration } & \multicolumn{11}{|c|}{ Instability number } \\
\hline & 1 & 2 & 3 & 4 & 5 & 6 & 7 & 8 & 9 & 10 & 11 \\
\hline CFG1 & 540.76 & 675.46 & 1573.4 & 2172.6 & 3441.2 & 3471.2 & 4133.1 & 4244.5 & 4608.8 & 5357.4 & 5769.6 \\
\hline CFG2 & 1976.2 & 3684 & 3838.9 & 4695.3 & 4944 & 5341.4 & 5934.9 & & & & \\
\hline CFG3 & 3466.4 & 3860.6 & 4231.8 & 4944.6 & 5273.6 & & & & & & \\
\hline CFG4 & 733.39 & 2994 & 3456.3 & 3892.7 & 4249.3 & 4267.5 & 4630.5 & 4937 & 5161.1 & & \\
\hline CFG5 & 645.31 & 1102.9 & 2543.3 & 3473.7 & 3711.3 & 3897.4 & 4939.6 & 5011.1 & & & \\
\hline CFG6 & 2447.9 & 2544.6 & 3477.2 & 3686.9 & 4121.6 & 4457.3 & & & & & \\
\hline CFG7 & 1877.2 & 3432.3 & 3654.5 & 4415.2 & 4692.8 & 5445.7 & & & & & \\
\hline CFG8 & 1859.8 & 3491.5 & 3816.3 & 4272 & 4705 & 4978.3 & & & & & \\
\hline CFG9 & 1974.1 & 3105.8 & 3474.6 & 4382.8 & 5140.8 & 5305.2 & 5802.7 & & & & \\
\hline CFG10 & 713.56 & 2315.4 & 3910.9 & 4415 & 4492.1 & 5244.3 & 5435.3 & & & & \\
\hline CFG11 & 1876.7 & 3889.7 & 4306 & 4500.7 & 4609.8 & 4869.1 & & & & & \\
\hline CFG12 & 1900.4 & 3809.6 & 4322.5 & 4608 & 4931 & 5863.9 & & & & & \\
\hline CFG13 & 698.1 & 2287.2 & 3459.3 & 3645.7 & 3862.1 & 4161 & 4946.9 & 539.91 & 5434.2 & & \\
\hline CFG14 & 3496.8 & 3814.6 & 4120 & 4733.2 & 5128.4 & & & & & & \\
\hline CFG15 & 1858.7 & 3431 & 3569.8 & 3851.5 & 4108.7 & & & & & & \\
\hline CFG16 & 1913.8 & 3459.3 & 3533.7 & 3810.5 & 4280.3 & 4790.2 & & & & & \\
\hline CFG17 & 1964.4 & 3775.5 & 4248.4 & 4357.5 & 4609.9 & 5277.6 & & & & & \\
\hline CFG18 & 1963.4 & 2742.6 & 3626.9 & 3800 & 4656.8 & 4890.6 & 4943 & 5334.7 & & & \\
\hline CFG19 & 1975.4 & 3643.2 & 3826.7 & 4691.5 & 4943.2 & 5337.1 & 5925.2 & & & & \\
\hline CFG20 & 669.19 & 1124.3 & 1882.5 & 4070.7 & 4653.5 & 5021.4 & & & & & \\
\hline CFG21 & 1881.4 & 2546.7 & 2560.6 & 2757.1 & 3677 & 4668.1 & & & & & \\
\hline CFG22 & 3407.6 & 3906.4 & 4361.3 & 4615.3 & 4941.6 & 5254.2 & & & & & \\
\hline CFG23 & 1970.3 & 3800.5 & 4552.6 & 4929.3 & & & & & & & \\
\hline CFG24 & 854.4 & 1851.8 & 3593.2 & 3759.1 & 4305.3 & 4689.9 & & & & & \\
\hline CFG25 & 1859.8 & 3491.5 & 3816.3 & 4272 & 4705 & 4978.3 & & & & & \\
\hline
\end{tabular}

A further study was carried out by comparing all modes (i.e. stable and unstable) of the different configurations and the results were similar. It points out two important conclusions: no correlation can be done between one unstable mode for a specific configuration and a stable mode of another configuration; and the whole modal basis is influenced by the contact states.

To investigate more precisely the problem, the MAC is also computed by considering just one component of the system. The different MAC matrix of each component are displayed Figure 8. For the sake of visibility, results are presented only for the first twelve configurations, but they are similar for the others. If only the disc is considered (see Figure 8 (a)), numerous correlations are observed. In other words the vibration modes of the disc are common to the different configurations. However, when a pairing can be done through the mode shape of the disc, it appears that there is no correlation between the other components, particularly in the case of the caliper and the bracket. These two components are highly sensitive to the contact states at the nine internal interfaces, and a pairing just by tracking the mode shapes of the disc could lead to a misunderstanding of the system behavior. To illustrate this observation, some mode shapes are represented and compared: for two different cases, the mode shapes of two modes which have a high MAC disc value are given in Figure 9 for the first case, and in Figure 10 for the second case. Their characteristics are given in Table 6 . For the first case, the unstable mode is located around $3480 \mathrm{~Hz}$ for configurations 5 and 14 . The global MAC is equal to 0.5748 , but the MAC associated to the disc is equal to 0.9555. Indeed, when the deformed shapes of the disc are compared, they are visually the same for both configuration. But if the focus is done on the caliper or on the bracket, the deformed shapes are different. It is confirmed by the two associated MAC values equal to 0.053 and 0.088 respectively. For configuration 5 (see Figure 9 (a) and (c)), there is almost no deformation on the bracket and maximum of amplitude are concentrated on the disc. But for configuration 14 (see Figure 9(b) and (d)), the bracket has an important deformation which is of the same order as the disc.

In the same way, by comparing the instability at $4250 \mathrm{~Hz}$ of configurations 3 and 4 , the disc has the same mode shape which is consistent with the high MAC value for the disc that is equal to 0.9444. But when looking more precisely the other components, their mode shapes are different. This is particularly true in the case of the bracket component, for which the MAC value is equal to 0.0423. For both configuration, the maximum of deformation is localized on the handle (see Figure 10). But for configuration 4, the deformation at the contact with the screw on the right is also important (see Figure 10(d)), whereas it is not the case for configuration 3 (see Figure 10(c)). 


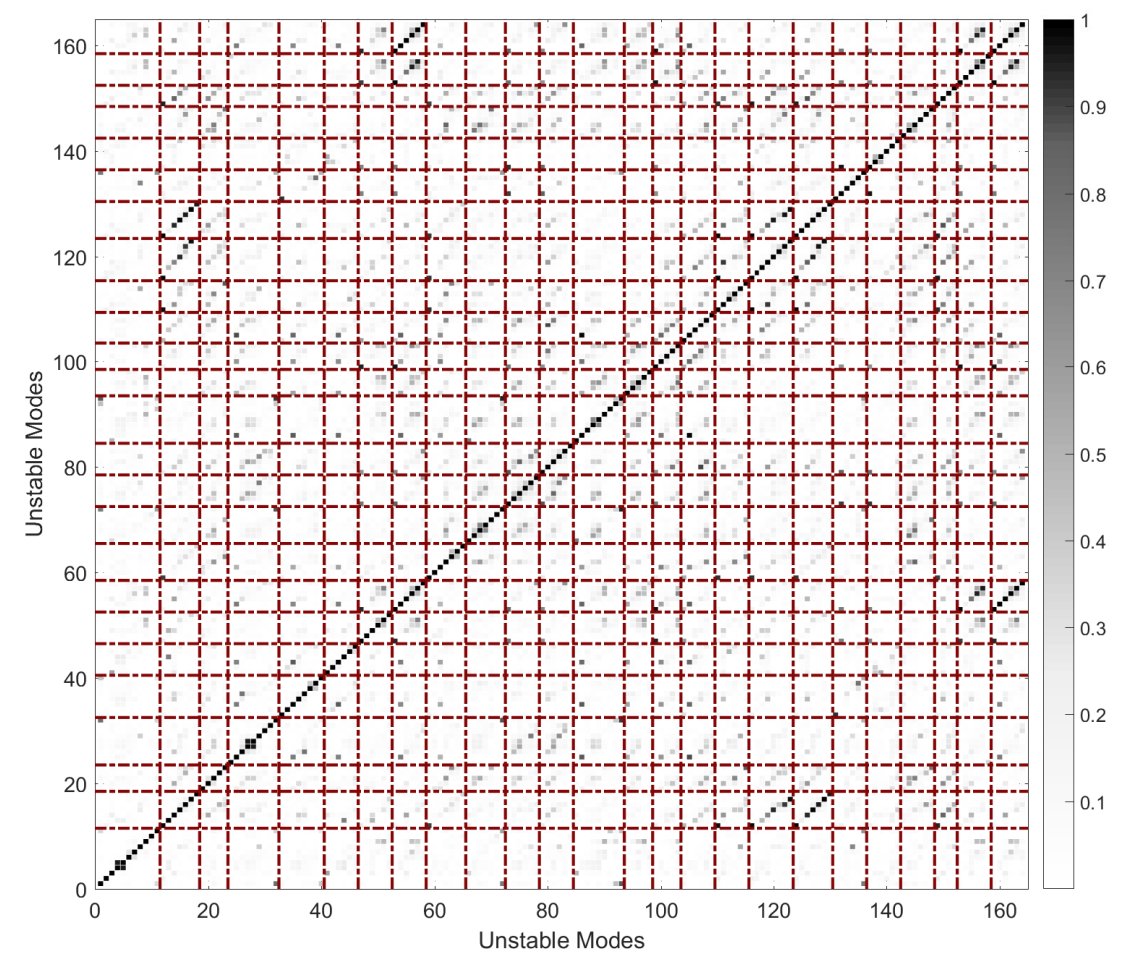

Figure 6: AutoMAC of the 25 configurations - Red dot lines corresponds to the separation between the configurations

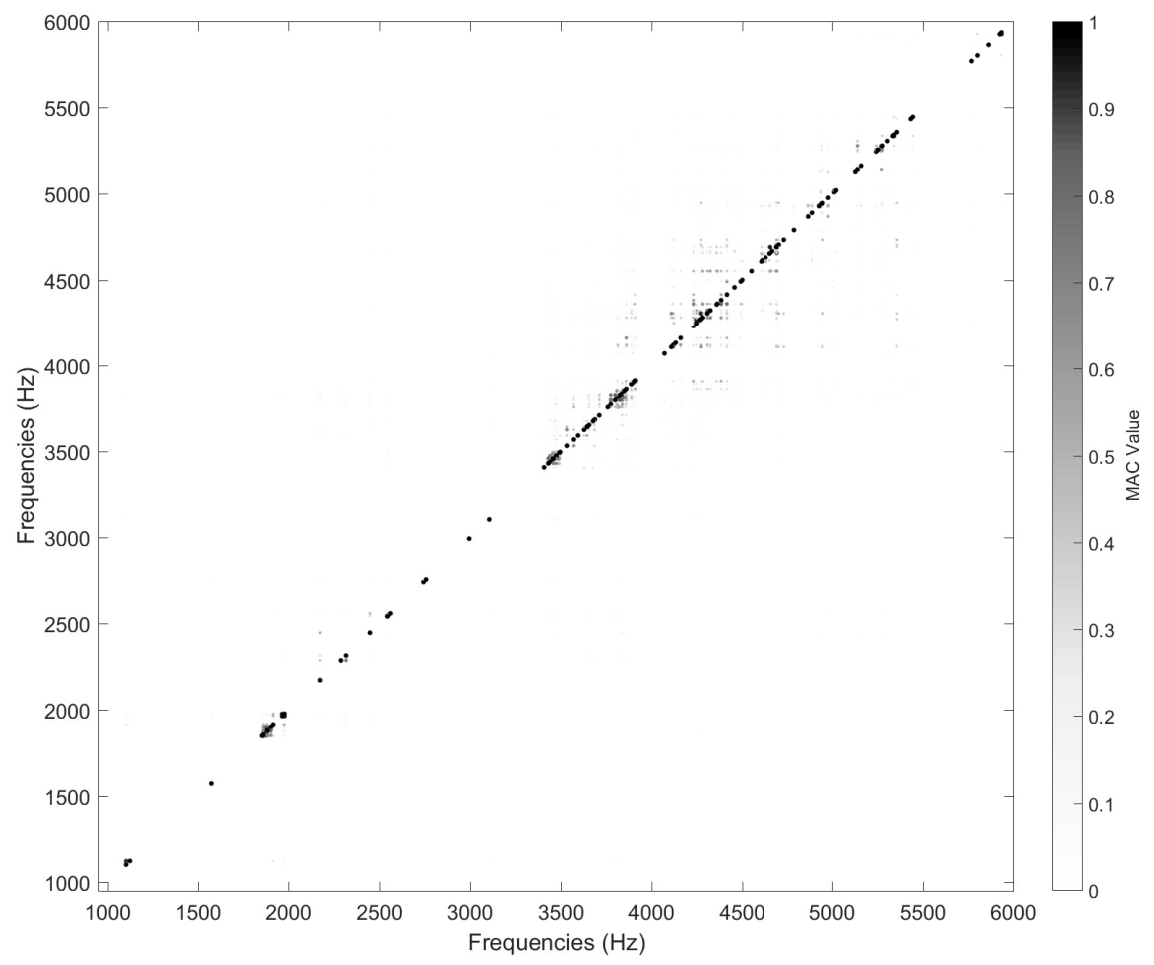

Figure 7: FAutoMAC of the 25 configurations - MAC values with a frequency scale 


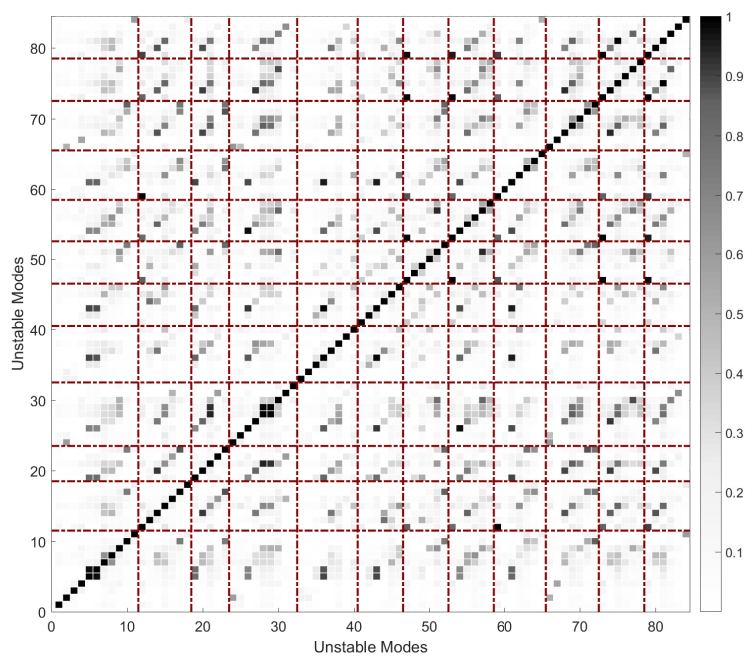

(a)

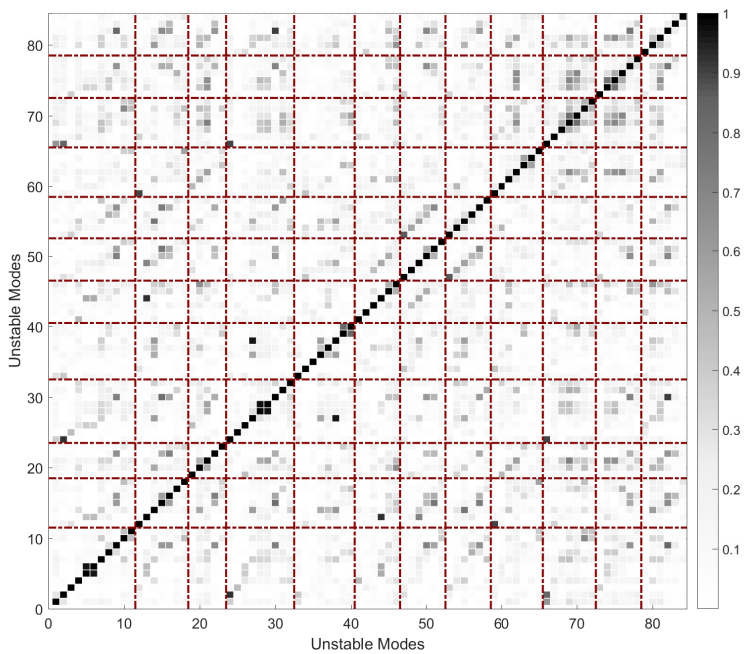

(c)

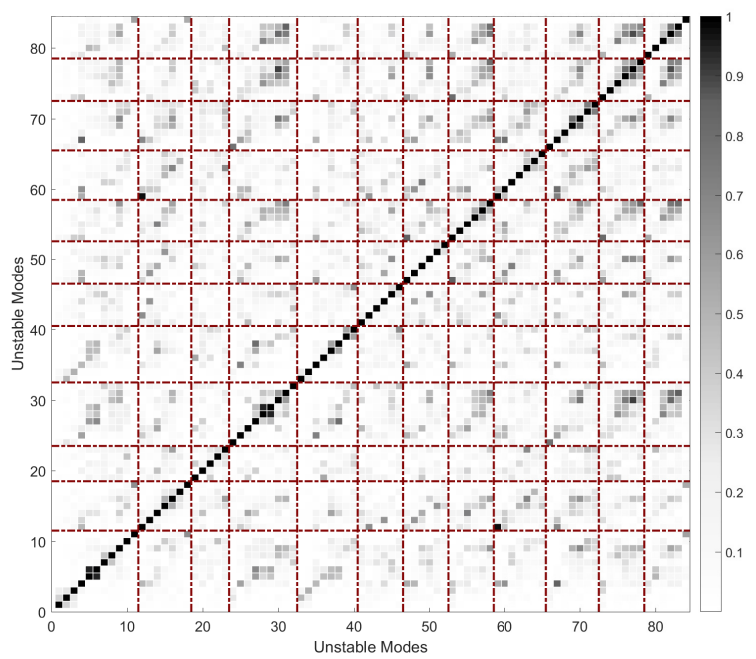

(b)

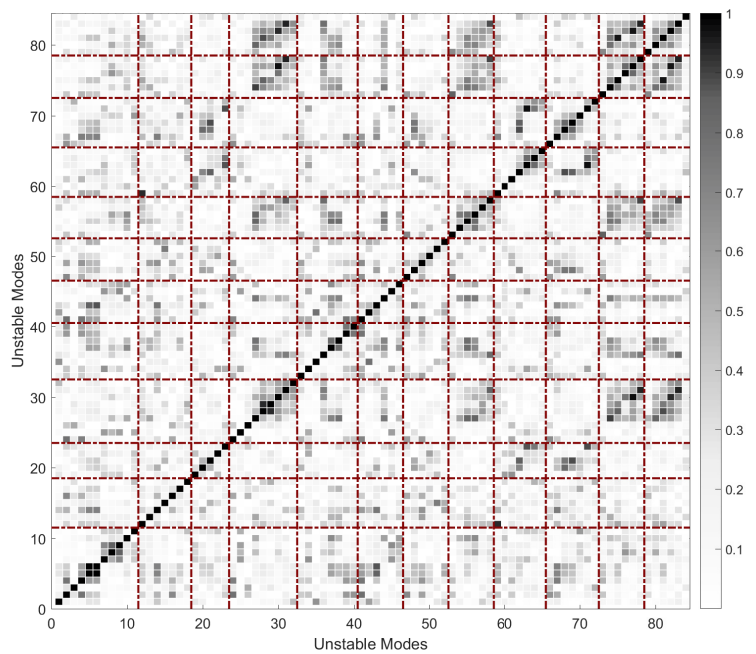

(d)

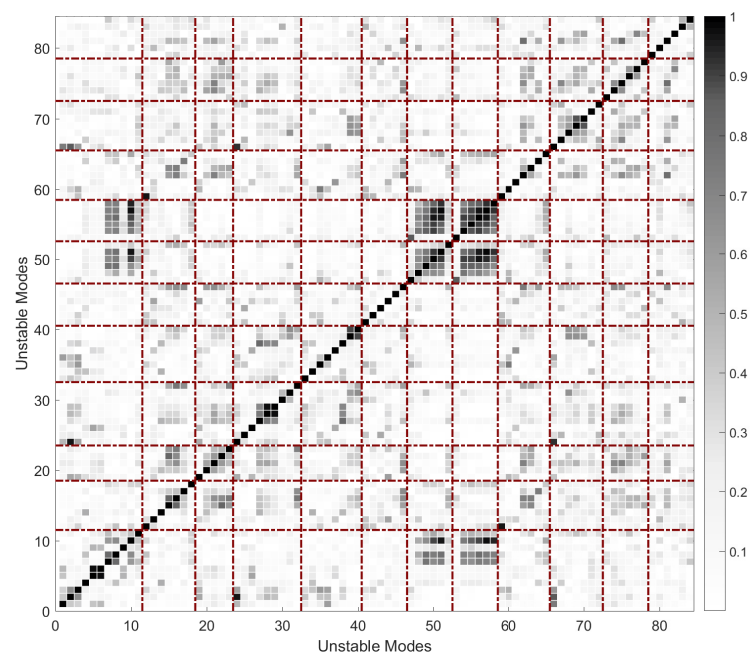

(e)

Figure 8: AutoMAC matrices of the unstable modes of 12 configurations for the different components: (a) Disc, (b) Caliper, (c) Bracket, (d) Inner Pad, (e) Outer Pad13 
Table 6: Characteristics of observed mode shapes

\begin{tabular}{lccccccc}
\hline Configuration & CFG5 & & CFG14 & & CFG3 & & CFG4 \\
\cline { 2 - 3 } \cline { 5 - 7 } Freq. (Hz) & 3473.7 & & 3496.8 & & 4231.8 & & 4267.5 \\
Real Part & 627.63 & & 302.73 & & 1429 & & 1376.7 \\
MAC Global & & 0.575 & & & & 0.371 & \\
MAC Disc & & 0.955 & & & & 0.944 & \\
MAC Bracket & & 0.053 & & & & 0.528 & \\
MAC Caliper & & 0.088 & & & & 0.042 & \\
MAC Inner Pad & & 0.126 & & & & 0.222 & \\
MAC Outer Pad & & 0.203 & & & & 0.566 & \\
\hline
\end{tabular}

In conclusion, these results lead to an important difficulty to deal with the different contacts conditions for the nine internal interfaces. It would have been interesting to identify one or several unstable modes common to the different configurations. Thus, the unstable eigenvalues could have been grouped together by tracking an unstable mode. However, the modes are sensitive to the contact states for each of the nine internal interfaces. So it is not possible to follow an unstable mode with respect to the different configurations. It is then necessary to adopt an other strategy to analyze the problem under study and to have an increased understanding of the role of each internal contact interfaces on the squeal propensity.

\subsection{Influence of the contact conditions per frequency range and restriction on the concept of the most unstable modes}

As it was previously shown, it is not possible to understand the role of each internal contact interfaces on the squeal propensity by tracking the unstable modes for all the frequency range of interest. In other words, being able to establish a relevant link between the appearance of instabilities via the mode shapes of instabilities and certain conditions of internal contacts is not easily achievable. So another strategy has to be adopted to analyse the problem under study and to better understand the role of each internal interface on the appearance of instabilities. In order to achieve such an objective, the automotive brake system is now studied for some specific frequency range of interest.

In order to illustrate the proposed approach, all unstable frequencies obtained with the 512 CEA are retained and the total frequency range $([0 ; 6000] \mathrm{Hz})$ is divided into sub-intervals of $5 \mathrm{~Hz}$. On each of these frequency intervals, the number of unstable frequencies present is determined, it corresponds to the occurrence of unstable frequencies displayed in Figure 11. Twelve small frequency intervals that focused on a peak of occurrences are retained, the main purpose being to establish whether some specific internal contact conditions are observable for a given peak of unstable frequencies. The characteristics of each frequency range are given in Table 7. Then, for each interval, the repartition of the contact conditions of the nine internal interfaces associated to the unstable frequencies are determined. Results are summarized in Figure 12 where, for each frequency range and for each internal contact, the percentage of repartition between a non-frictional state (in blue) and a frictional state (in yellow) is given. For a few cases, the role of some internal contact states in the existence of the instability of a frequency range of interest is obvious. For example, under $1140 \mathrm{~Hz}$, the existence of instabilities is always related to a non-frictional state of the contacts DB and DH. For the frequency range $[1400 ; 1470] \mathrm{Hz}$, instabilities are mainly related to a frictional state of contacts DB, PP and RHI and a non-frictional state of contacts DH, RHE, RCBE and RBE. For $[1150 ; 1650] \mathrm{Hz}$, the emergence of instabilities corresponds to a non-frictional state of contacts RBE and RCBE. However, if such an analysis gives some interesting tendencies in some cases, there is no concrete conclusion for most frequency ranges. See for instance the frequency ranges $[1800 ; 2000] \mathrm{Hz}$ and $[3775 ; 3930] \mathrm{Hz}$ where the repartition is around $50 \%$ for each internal contact interface.

This approach is interesting but seems limited since it is possible to find two completely different mode shapes with similar natural frequencies. Moreover, the results are strongly influenced by the choice of the frequency range, which can be difficult to determine when the peaks of the occurrences of unstable frequencies are not clearly distinct. For this reason a more comprehensive approach must be defined. Instead of characterizing the condition of instabilities existence, the choice is made to characterize the most unstable configuration of larger frequency ranges, which can be assume at a first glance as the worst case scenario for an automotive brake system. This choice can be interpreted as a simplification or a restriction of the general problem to the study of the influence of internal contact for the most unstable modes.

This leads us to identify the contact state of each of the nine internal interface that brings the system in the most unstable configuration. The notion of the "most unstable configuration" is not necessarily unique and 

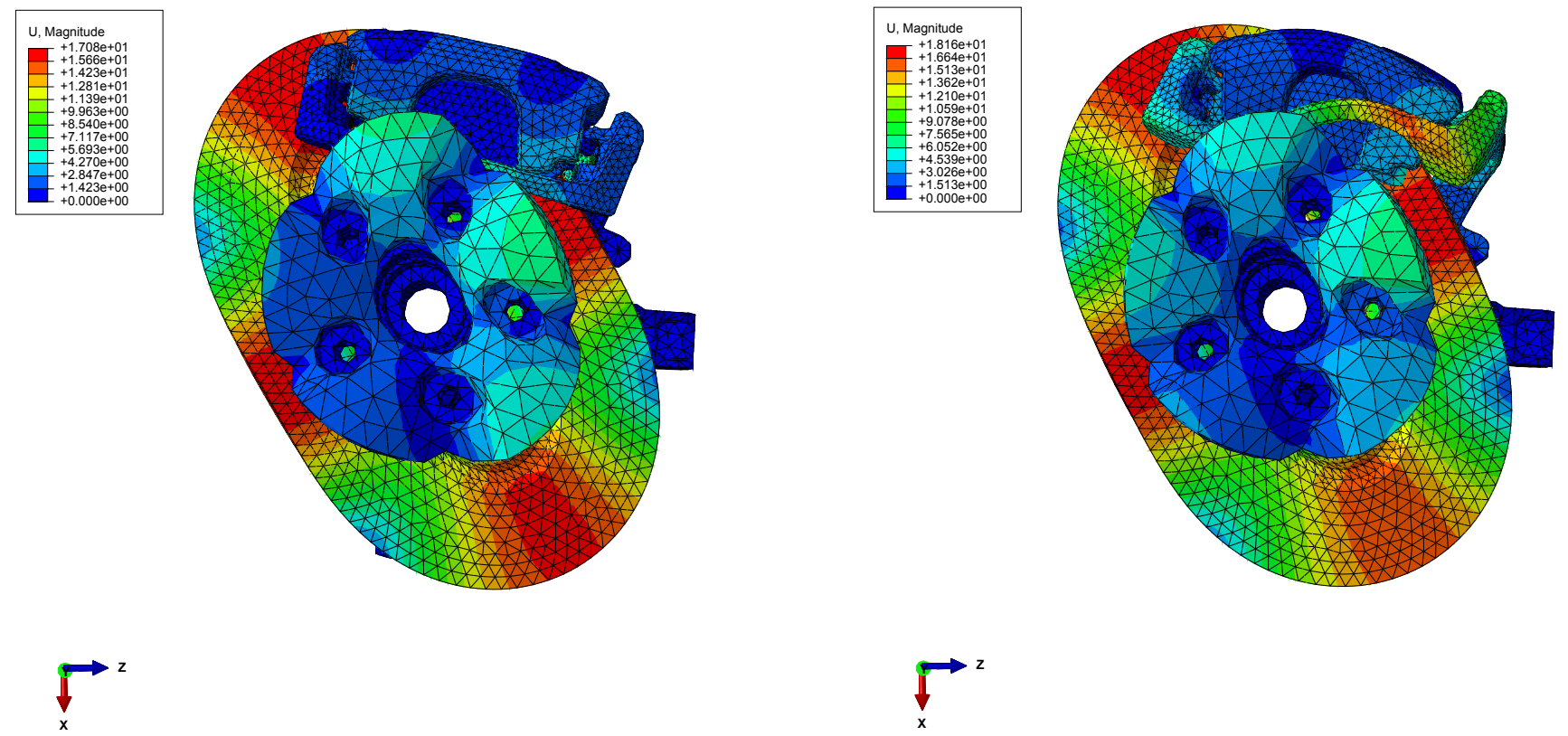

(a)
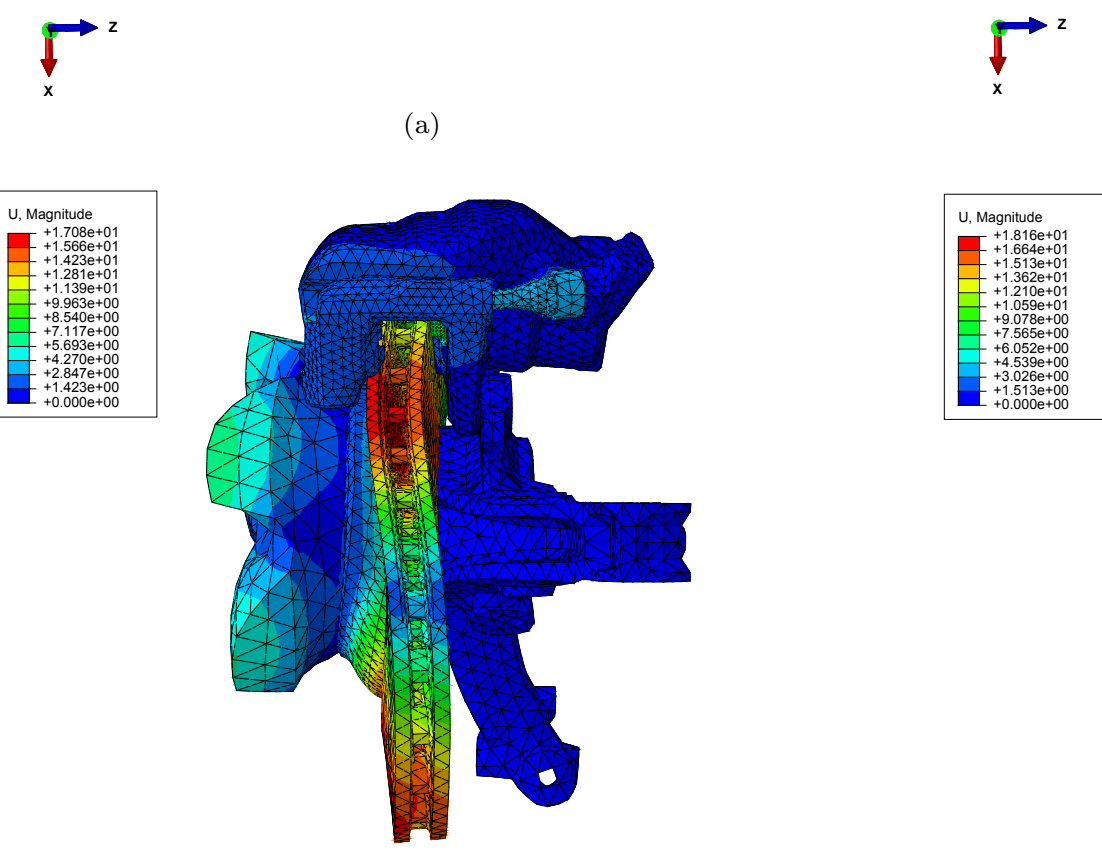

(b)
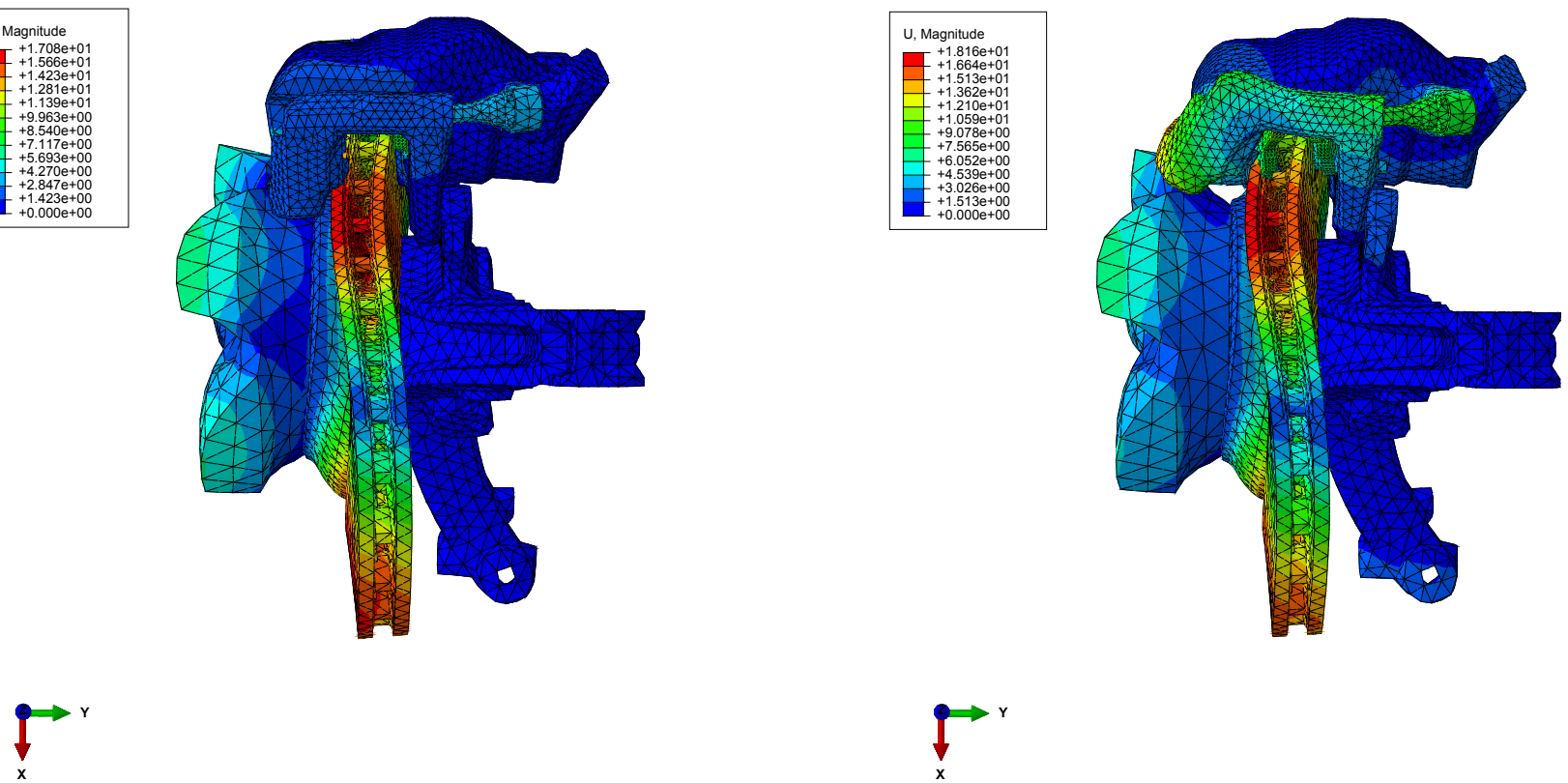

(c)

(d)

Figure 9: Comparison of mode shapes of configurations 5 (left) and 14 (right) - Face view (top) and Profile view (bottom) 

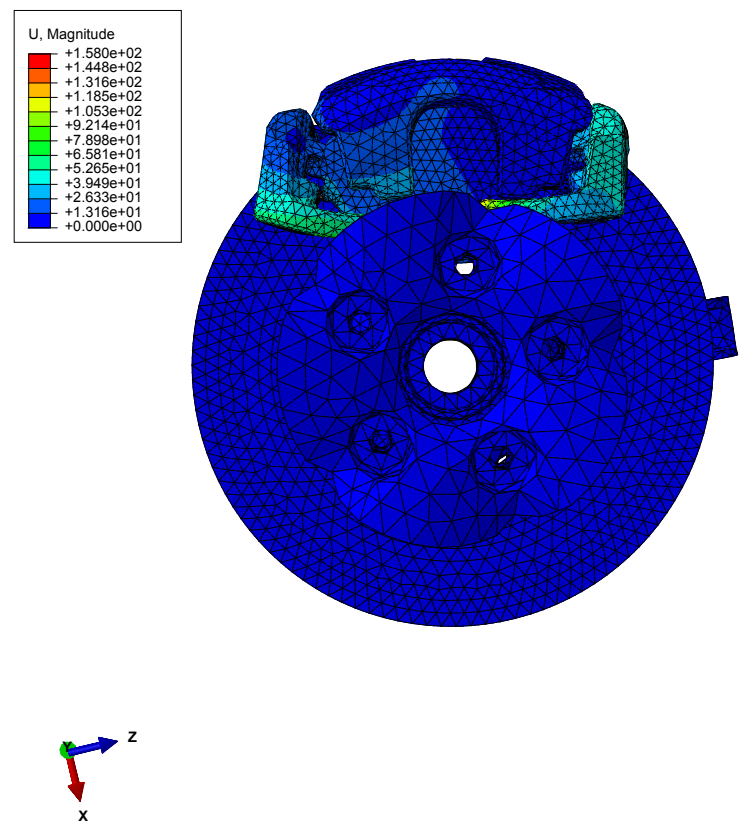

(a)
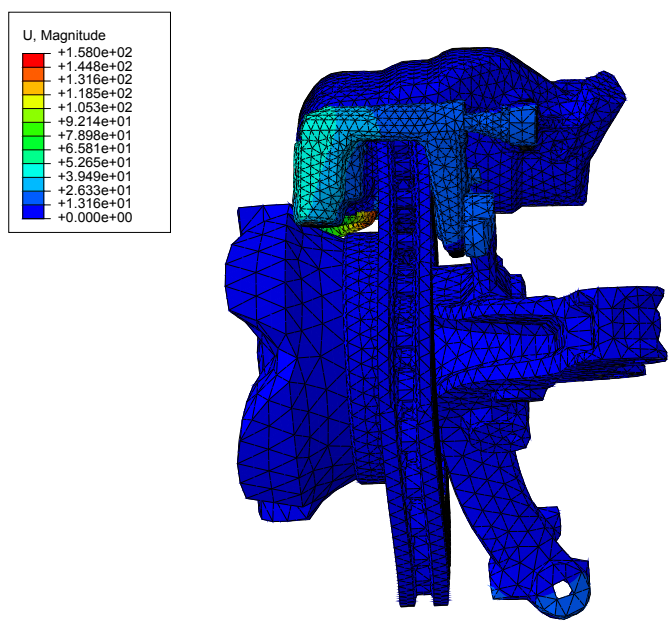

$\underset{x}{\longrightarrow}$

(c)
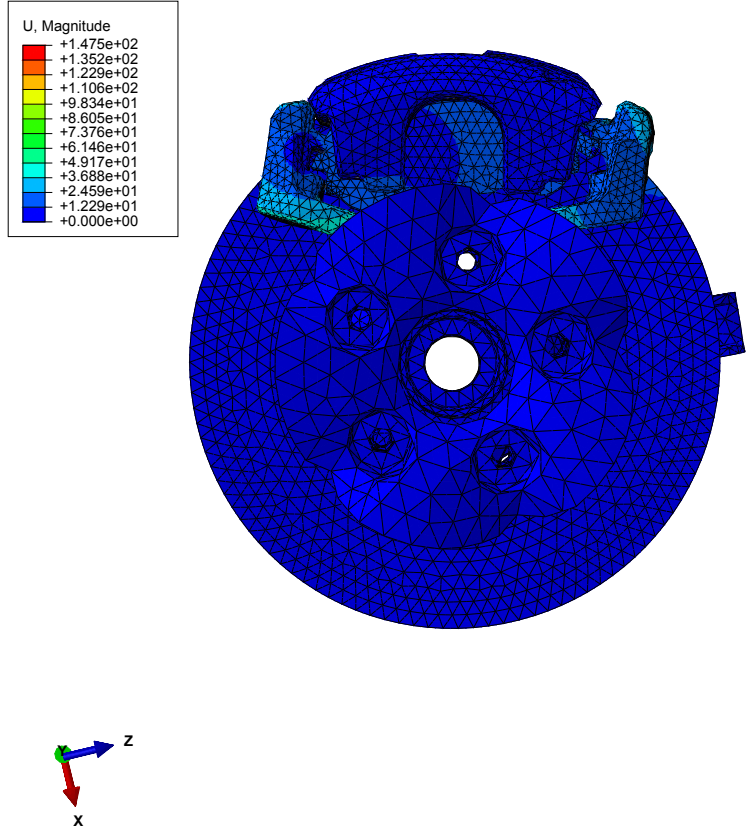

(b)
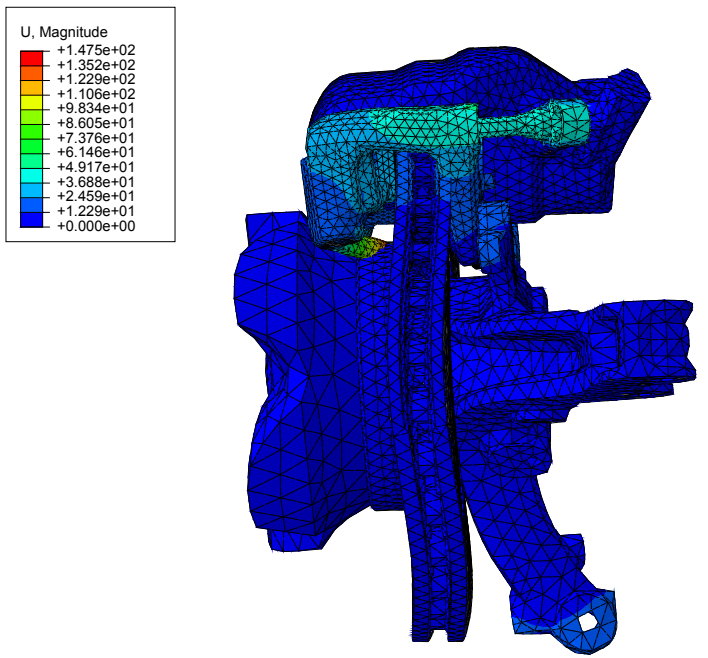

(d)

Figure 10: Comparison of mode shapes of configurations 3 (left) and 4 (right) - Face view (top) and Profile view (bottom) 


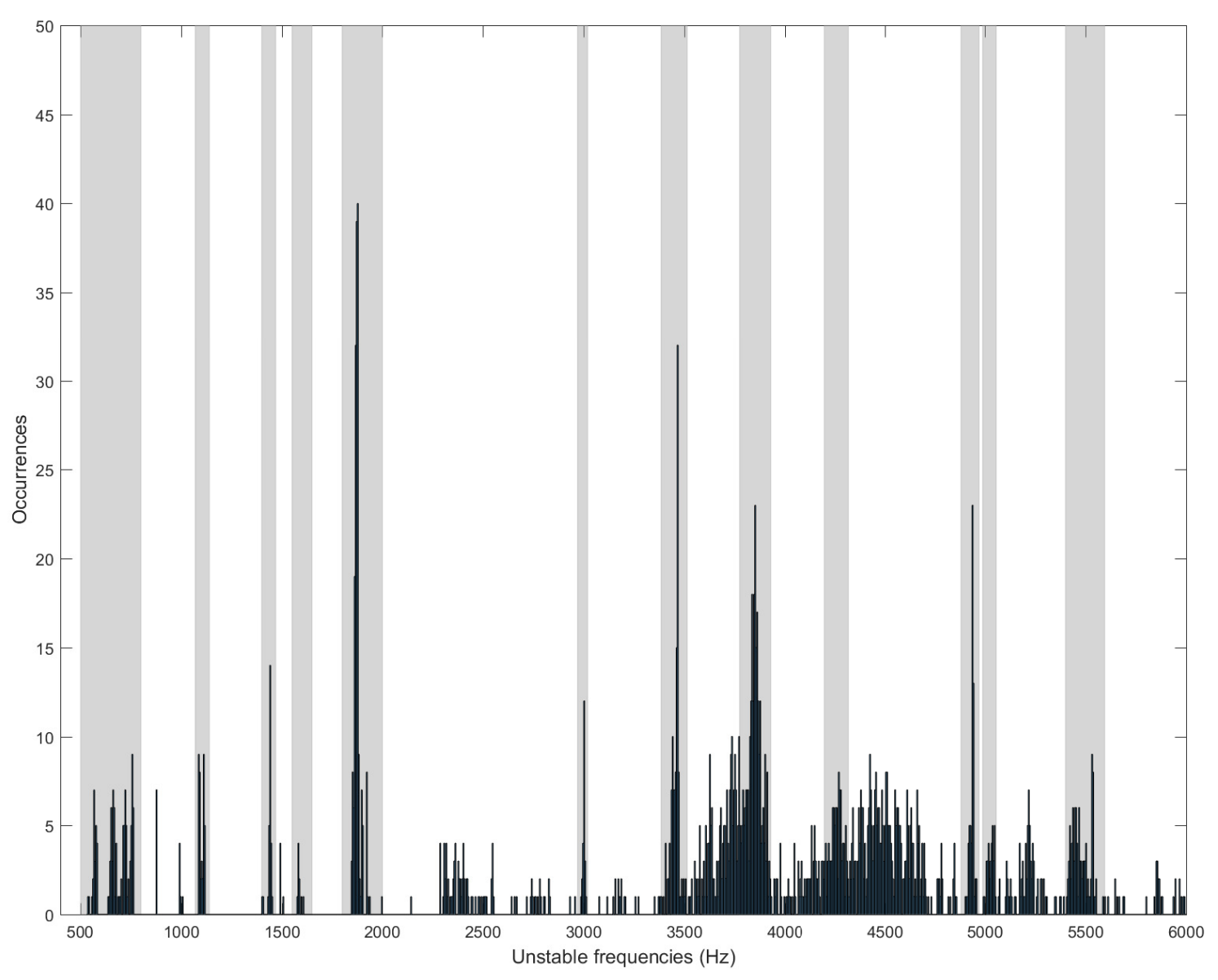

Figure 11: Occurrences of unstable frequencies and intervals of study

universal. Here, the most unstable configuration is defined by the configuration with the maximum real part (i.e. the highest growth rate of the unstable mode). It is worth to remind that, because the analysis is based only on the CEA, this "most unstable configuration" does not mean necessarily the configuration that induces the most important squeal noise in the brake system but the configuration with high possibility of squeal noise. It is worth to remind that the stability analysis may lead to an underestimation or an overestimation of the unstable modes as previously explained in Section 2.1 and a complete nonlinear simulation should be performed in addition to the stability analysis to estimate the real most unstable configuration in regard to squeal noise. This is out of reach of the present study because of the numerical implementation difficulty and the prohibitive calculation times associated with the estimation of friction-induced vibration and noise for a FEM brake system. The frequency range under study is reduced to $[0 ; 6000] \mathrm{Hz}$ in order to be concise in our analysis, the idea being to illustrate the relevance of the proposed methodology.

Then the methodological approach is the following one: the status of one internal contact interface is imposed (a frictional state or not) while all other internal interfaces can be in any state. Then $2^{8}=256$ CEA are performed and the maximum of real parts is determined. This procedure is repeated for each of the nine internal contact interfaces by applying the non-frictional state (with $\mu_{\text {intern }}=0$ ) or friction (with $\left.\mu_{\text {intern }}=0.15\right)$. Because some configurations are redundant, only $512 \mathrm{CEA}$ calculations are required. The results obtained with $\mu_{\text {pad-disc }}=0.3$ and $\mu_{\text {pad-disc }}=0.5$ are presented in Figure 13 .

A relevant analysis of the impact of each internal contact is then possible. For example, if the contact between the piston and the inner pad is non-frictional (see Figure 13b at contact PP and blue point), the maximum real part is equal to 725 , while it is equal to 1100 in the case of frictional state for PP is (see yellow point). Here the "most unstable configuration" versus the contact PP corresponds to the frictional state.

More generally, it also appears that the two internal contacts that give the most important difference of results (in terms of the Maximum Real Part) according to their state of contacts (i.e. frictional or not state) 
Table 7: Frequencies intervals

\begin{tabular}{cc}
\hline $\mathbf{N}^{o}$ & Freq. interval $\mathbf{( H z )}$ \\
\hline $\mathbf{1}$ & {$[500 ; 800]$} \\
$\mathbf{2}$ & {$[1070 ; 1140]$} \\
$\mathbf{3}$ & {$[1400 ; 1470]$} \\
$\mathbf{4}$ & {$[1550 ; 1650]$} \\
$\mathbf{5}$ & {$[1800 ; 2000]$} \\
$\mathbf{6}$ & {$[2970 ; 3020]$} \\
$\mathbf{7}$ & {$[3385 ; 3515]$} \\
$\mathbf{8}$ & {$[3775 ; 3930]$} \\
$\mathbf{9}$ & {$[4200 ; 4320]$} \\
$\mathbf{1 0}$ & {$[4880 ; 4970]$} \\
$\mathbf{1 1}$ & {$[4988 ; 5055]$} \\
$\mathbf{1 2}$ & {$[5400 ; 5595]$} \\
\hline
\end{tabular}

are the piston-inner pad contact (PP) and the inner top radial contact (RHI). On the contrary, the bottom and top caliper-pad contact (DB and DH) give very close results (in terms of the Maximum Real Part) regardless of their contact status. These results indicates that considering the possibility of a change of contact status for the two internal contacts DB and DH is not necessary for a future analyze and design studies. Moreover according to the level of accuracy required in a design process, it is also possible to define the internal contacts whose two states (friction or not) to be kept and those for whom a contact state is privileged. For example, if the most unstable scenario has to be characterized at about $5 \%$, then it is characterized by a frictional state for internal contacts PP, RHE and RBI and by a non-frictional state for internal contacts RHI, RCBI, RCBE and RBE. The state of the two internal contacts DB and DH is in the margin of error.

Now the same analyze can be carried out for different frequency ranges in order to better understand the role of each internal interface on the appearance of instabilities by frequency ranges of interest. Results are displayed in Figure 14. As in the previous case, it is possible to create a hierarchy between the different internal contacts and to decide whether or not to retain the possibility of the two state for a specific internal contact in a design process. It is also observed that the internal contacts that appear to be the most influential on the "most unstable configuration" depend of the considered frequency range. For instance, the contribution of the contact PP is low for $[950 ; 2005] \mathrm{Hz}$ but crucial for $[4000 ; 4890] \mathrm{Hz}$. On the contrary, the contribution of the two contacts DB and DH is high for the two frequency ranges $[0 ; 950] \mathrm{Hz}$ and $[950 ; 2005] \mathrm{Hz}$ but low between $[4000 ; 4890] \mathrm{Hz}$. It is also noted that the influence of the internal contact states DB and DH versus the "most unstable configuration" are reversed on the first two frequency ranges. For the first frequency range $[0 ; 950] \mathrm{Hz}$ the "most unstable configuration" is obtained for the non-frictional state of the internal contacts DB and DH, whereas it corresponds to the friction state of DB and DH for the second frequency range [950;2005] Hz.

In conclusion, the proposed strategy allows us to rule on the effect of all independent internal contacts. Some internal contacts have a large contribution and play a key role on the propensity of automotive brake squeal, whereas the others have a small impact and only add a slight variation on the instability phenomena. Moreover it is possible to propose a restriction of the potential evolution of the contact states to be considered for each internal interface according to a given design process versus a frequency range of interest. Considering that the CEA enables to get the squeal propensity but has the disadvantages of being under- or over- predictive, the determination of this area and its characteristics may be enough in a design process.

\section{Use of a genetic algorithm to determine the most critical scenario}

\subsection{Motivation for an industrial point of view}

This section is devoted to the use of a Genetic Algorithm (GA) to identify the case scenario defined as "the most unstable configuration". Finding this type of configuration is indeed an objective of interest for the PSA automotive industry. This may seem paradoxical since one would expect to look for the design leading to the least squeaky system. However, the prohibitive cost of calculation makes it impossible to propose a global design strategy that includes the internal interfaces. So the proposed procedure is divided into two steps. First, because the actual behavior of these internal contact interfaces in service is uncertain and not controlled, the most harmful design in regard to them is determined. And finally, a design study on controllable parameters is performed on this "most unstable configuration" in order to mitigate it. For example, the selected instability in 

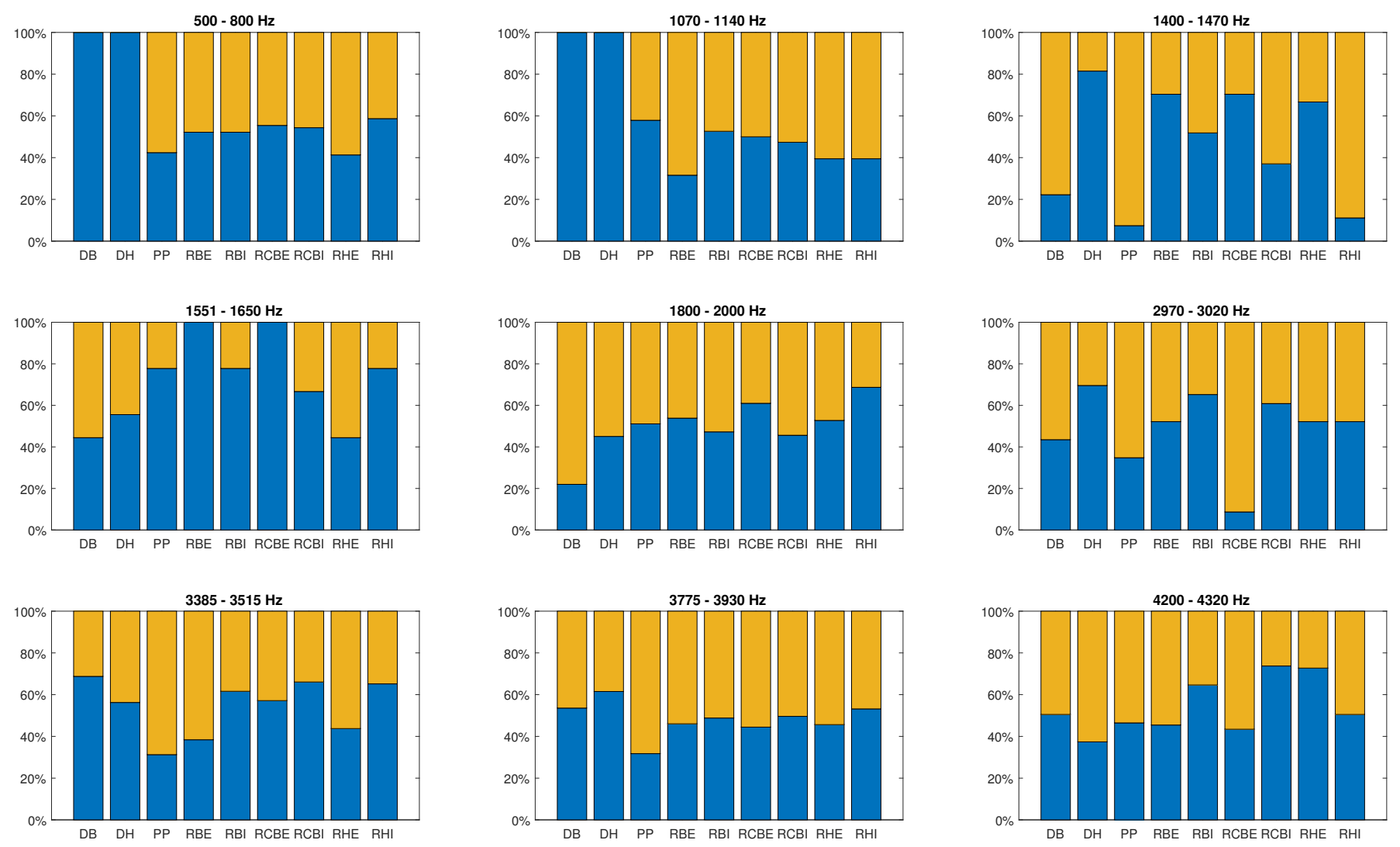

Figure 12: Percentage of repartition of contact states for the different frequency intervals - Blue: non-frictional state $\left(\mu_{\text {intern }}=0\right)$ and yellow: frictional state $\left(\mu_{\text {intern }}=0.15\right)$

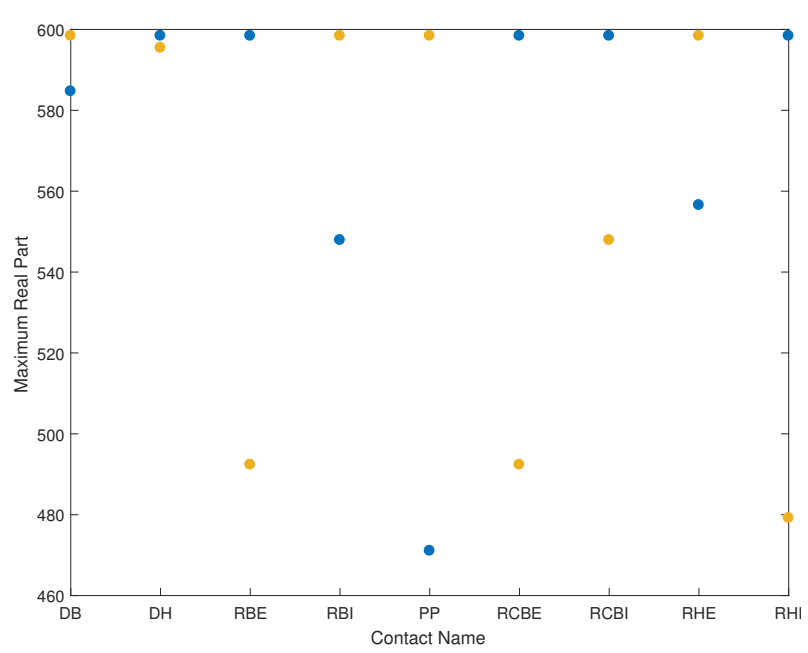

(a)

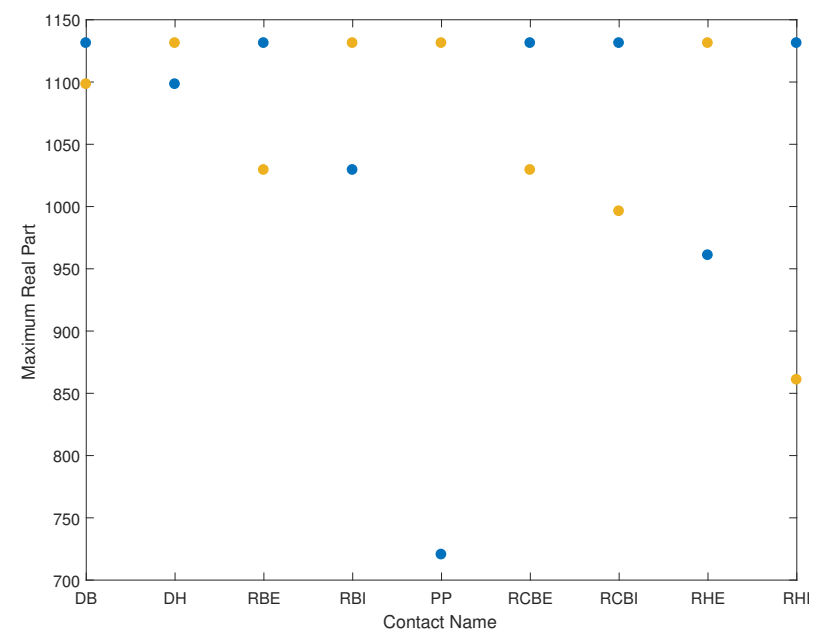

(b)

Figure 13: Maximum of real part assuming a contact condition - Blue for a non-frictional state $\left(\mu_{\text {intern }}=0\right)$ and yellow for a frictional state $\left(\mu_{\text {intern }}=0.15\right)$ - for $\mu_{\text {pad } / \text { disc }}=0.3$ (top) and $\mu_{\text {pad } / \text { disc }}=0.5$ (bottom) 

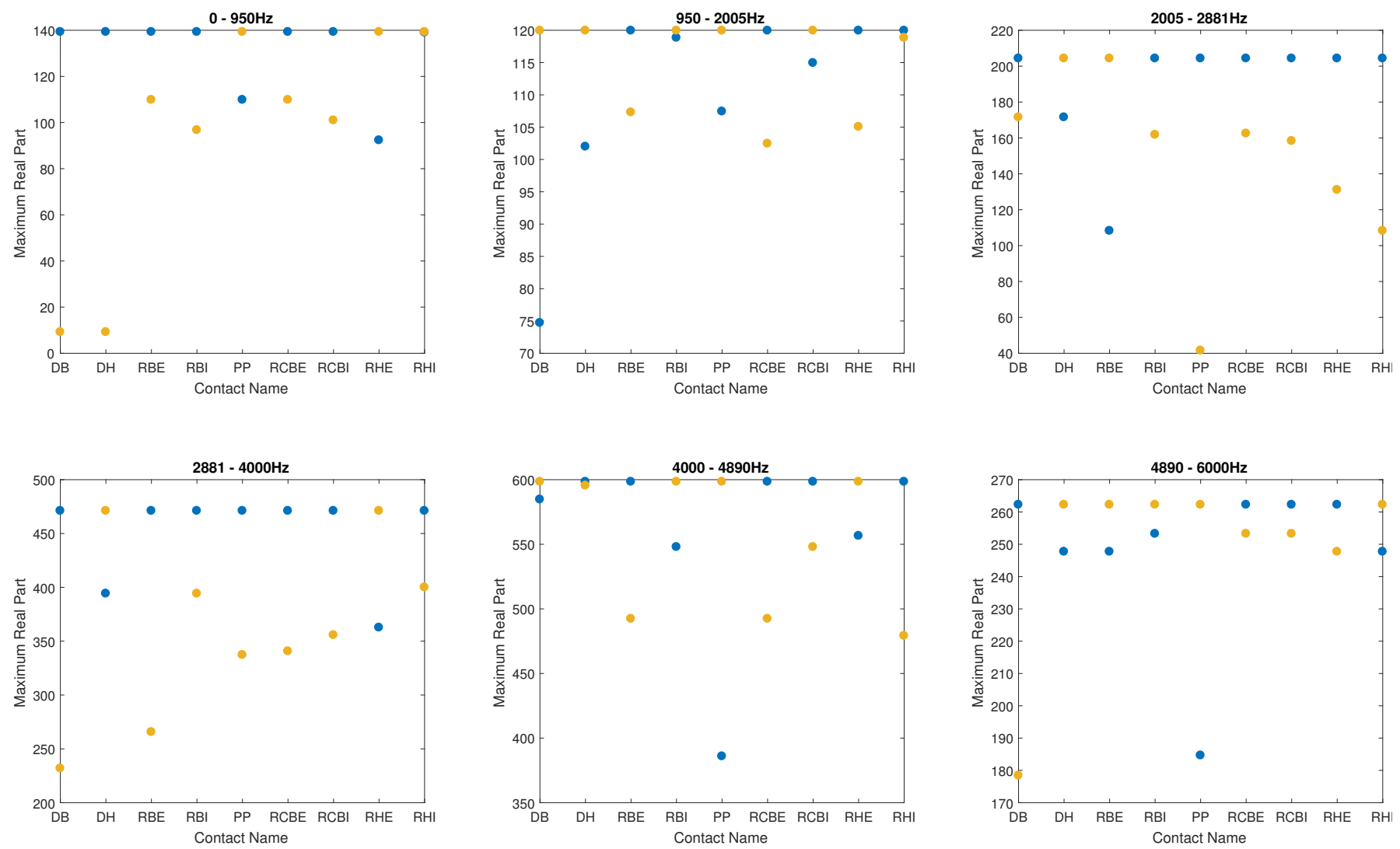

(a)
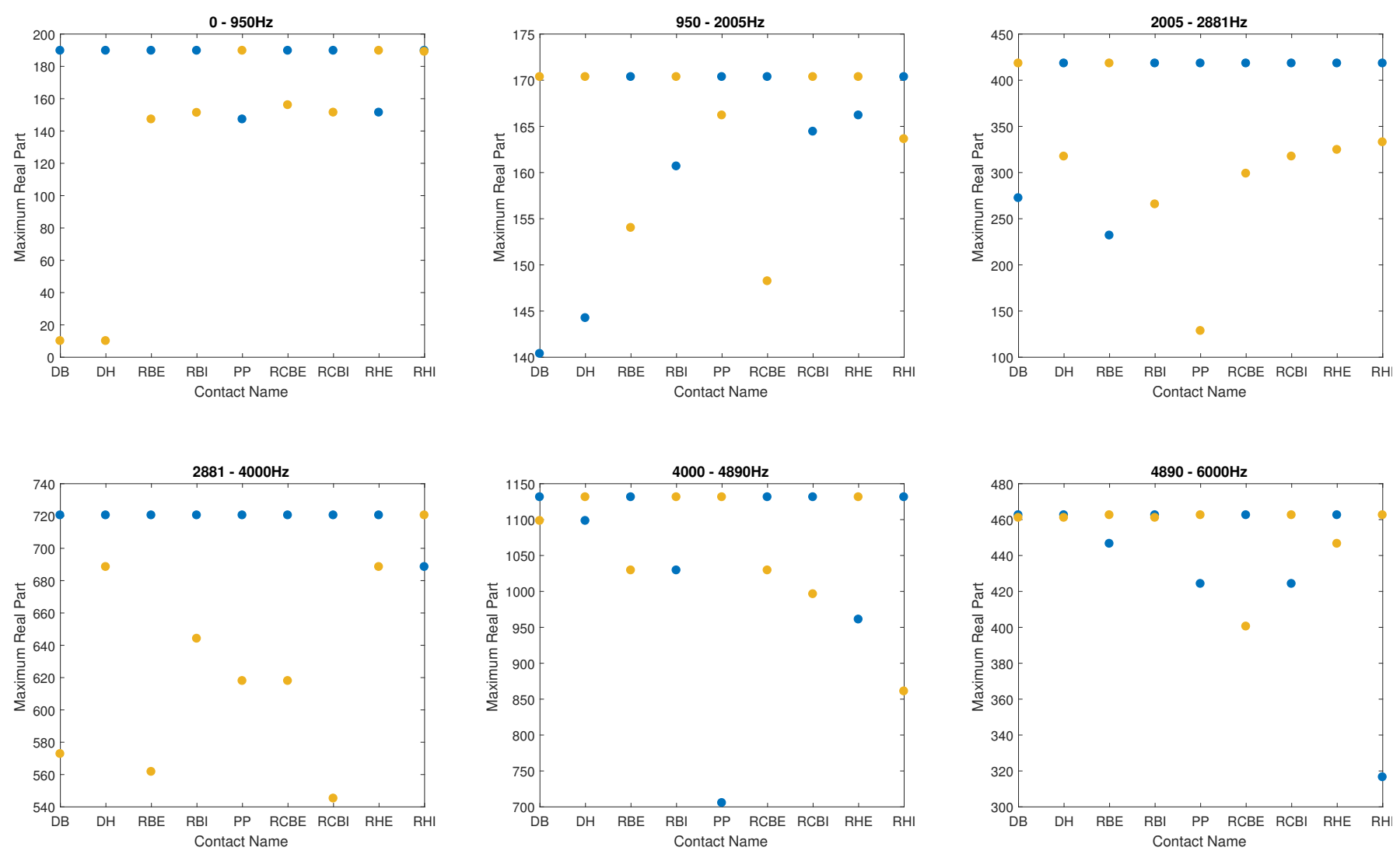

(b)

Figure 14: Maximum of real part assuming a contact condition per frequency range - Blue for a non-frictional state $\left(\mu_{\text {intern }}=0\right)$ and yellow for a frictional state $\left(\mu_{\text {intern }}=0.15\right)$ - for $\mu_{\text {pad } / \text { disc }}=0.3$ (top $)$ and $\mu_{\text {pad } / \text { disc }}=0.5$ (bottom) 
Table 8: Frequencies intervals

\begin{tabular}{cc}
\hline $\mathbf{N}^{\mathbf{o}}$ & Freq. interval $\mathbf{( H z )}$ \\
\hline 1 & {$[950 ; 2005]$} \\
2 & {$[2005 ; 2881]$} \\
3 & {$[2881 ; 4000]$} \\
4 & {$[4000 ; 4890]$} \\
5 & {$[4890 ; 6000]$} \\
\hline
\end{tabular}

the automotive brake system design should then be eliminated or reduced by changing the geometry or material properties of the brake components to decouple the coupling modes.

To cope with the high number of internal contacts and the too high numerical cost of taking all configurations into account, a first reduction of the problem size is realized by only assuming two different contact states for each internal interface: non-friction with $\mu_{\text {intern }}=0$ or friction with $\mu_{\text {intern }} \neq 0$, and the variation of the friction coefficient $\mu_{\text {intern }}$ for $\mu_{\text {intern }} \neq 0$ is not taken into account (this assumption is in agreement with the first conclusions of the study). However, it still represents a high number of CEA to perform since there are numerous internal contacts, so it is necessary to find a strategy to overcome this numerical cost. Because the problem is discontinuous, a strategy based on GA is undertaken.

\subsection{Genetic algorithm and adaptation for the problem under study}

This section aims to briefly recall the concept and approach associated with Genetic algorithm (GA) that is a kind of Evolutionary Algorithm (EA) used to approximate the solution of a given problem in a reasonable time. In this study, 6 objective functions are considered and correspond to the maximal real part for each frequency range provided in Table 8 . A population, in which the individuals try to improve themselves evolves, generation after generation with respect to the objectives. An individual is a configuration and is described by its genes (i.e. the status of the different internal contacts) and is associated to the maximal real part observed on each frequency range. A population is a group of individuals (i.e. a group of configurations). A generation is an iteration of the algorithm and corresponds to the current population. The general methodology is sketched in Figure 15(a). The different steps are the following:

- Step 1: Initialization with an initial group of configurations created randomly,

- Step 2: Evaluation of maximal real parts of each configuration,

- Step 3: Creation of new configurations by selection, crossover and mutations,

- Step 4: Evaluation of maximal real parts of each configurations,

- Step 5: Creation of a new population with old and new configurations.

Steps from 3 to 5 are iterated until a stop criterion is reached.

The different operators that characterized the GA are :

- Selection: different strategies are proposed in the literature as the wheel selection, the tournament selection or even the elitist selection. In the present study, the tournament selection is used. It consists in the creation of tournaments between individuals (i.e. configurations) chosen randomly in the population and the best of them are selected.

- Crossover: this operator is used to create the genes (i.e. contact status) of the new generation from those of the previous one. Several techniques are possible as the single-point crossover, the two-points crossover or the uniform crossover. Here, a uniform crossover is considered. For each contact, the child's contact status is chosen randomly between one of the two parents.

- Mutation: this operator creates random modifications of genes (i.e. of contact status). This is important for GA to replace "forgotten" genes (i.e. contact status) of old population or to test new combinations.

For more details, the reader can refer to [31, 32]. GA are adapted to both Single-Objective (SO) optimization and Multi-Objective (MO) optimization and according to the application, different methods exist. For the present study, the objective is to determine the internal contact status that give the maximum real part on each frequency range of interest. Since there are 6 frequency ranges an so 6 objective functions, a MO algorithm 


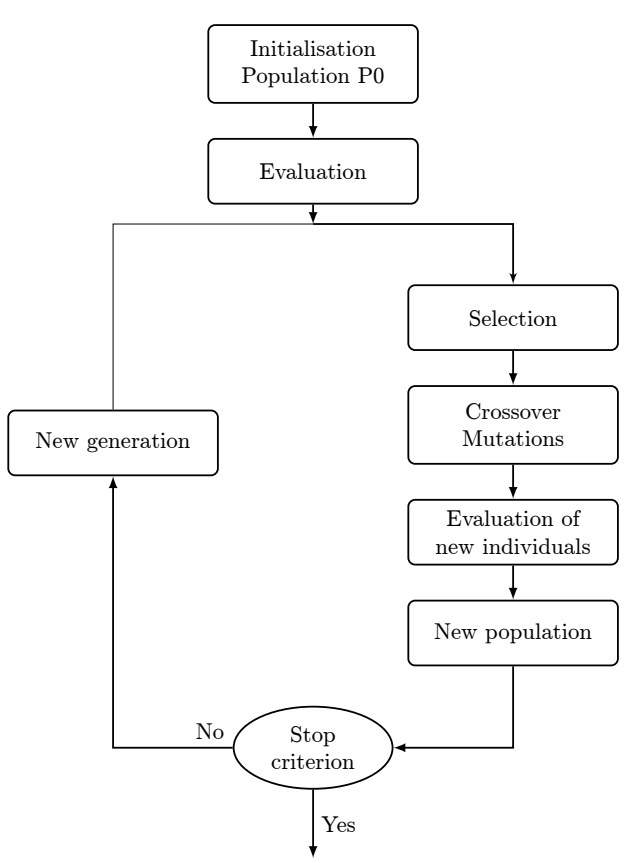

(a)

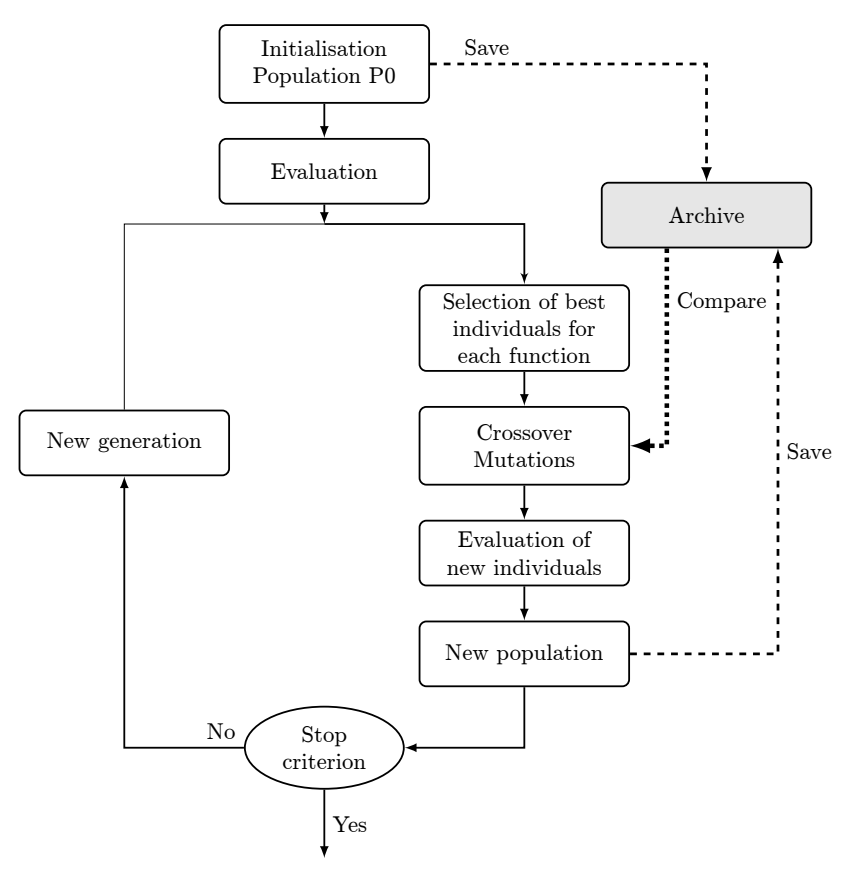

(b)

Figure 15: Diagrams of algorithms: genetic algorithm (a) and multi-population genetic algorithm (b)

may be conceivable. However, a MO-GA determines the Pareto front of the different objective functions, which is not what we are looking for in our study. In fact, our goal here is to determine the solution (i.e. "the most unstable configuration") for each frequency range. So the strategy consists to launch a SO-GA for each function. If they started one after the other, the different populations cannot learn from each other. Also the proposition developed here relies in the launch of the six SO-GA simultaneously with a common population. The general sketch of the method is given in Figure 15(b). Overall operation remains the same: crossover, mutations and child evaluations are realized for the global population. The difference relies in the selection of parents: the whole population is considered and the $N / N_{f}$ best individuals for each objective functions are selected for the next step, where $N$ is the number of parents to select and $N_{f}$ the number of objective functions. In order to limit the number of CEA to perform, an archive is created with all tested individuals and new individuals must not be inside.

\subsection{Numerical results}

Two different sets of parameters are considered for the GA. They are given in Table 9. The first set is characterized by a smaller population and a low number of potential parents but more generations are accepted, when the second set has a bigger population and a higher number of potential parents but the maximal number of generations is smaller. To compare the performances of the two sets, each algorithm is launched 1000 times.

An example of obtained results is displayed in Figure 16 for both set of parameters. The different individuals tested by the algorithm are in black, the solution obtained by the algorithm in blue and the theoretical solution in red. It is clear that the algorithm gives a good approximation of the reference solution and the map created by the different individuals gives a good overview of the complete complex plan (compare to Figure 5).

To evaluate the robustness of the algorithm, the percentage of cases where the correct solution is estimated in a certain margin of error is displayed for each function (see Figure 17). In both cases, solutions are well approximated except in the case of the third frequency range. This latter case is particular. Indeed considering reference results, if one considers the second eigenvalue closest to the solution for this frequency range, then there is $22 \%$ of difference between the real parts. For the other frequency ranges, the algorithm proves its efficiency and gives a satisfactory estimation. If a margin of error of $10 \%$ is considered, the second set of parameters has better results than the first one. For example, between $[950 ; 2005] \mathrm{Hz}$, with the first set the solution is estimated with less than $10 \%$ of error in $75 \%$ of cases, whereas it is in $90 \%$ of cases for the second set of parameters. It can be noted that if a margin of error of $20 \%$ is considered, then their performances are very similar.

The repartition of the contact status of GA solutions is also displayed in Figure 18. Results are very similar for both sets of parameters and some tendencies appear. For example, for the second frequency range (between 
Table 9: Genetic algorithm parameters

\begin{tabular}{lll}
\hline $\mathbf{N}^{o}$ & $\mathbf{1}$ & $\mathbf{2}$ \\
\hline Population size & 25 & 30 \\
Number of selected individuals & 12 & 20 \\
Number of generations & 5 & 4 \\
Average number of calculations & 86.3 & 122.8 \\
\hline
\end{tabular}

[950; 2005]Hz), GA solutions always have a frictional state for contacts DB, DH and almost RBI; and always a non-frictional state for contacts RCBE and almost RBE. These results have to be compared with results presented in Figure 14. For the second frequency range, the most influential contacts are DB, DH, RCBE and RBE. It appears here that the GA has identified quickly the most influential contacts on the frequency range. In the same way, similar analysis can be made on the other frequency ranges for different contacts.

From a computational cost point of view, the second set of parameters requires 36 extra calculations compared to the first set. These extra calculations bring a better precision on the estimation of the real part for each frequency and for all the margin of error calculated; however, they do not bring real contribution on the identification of the most influential contacts. In fact, the main influential contacts are determined in a few generations (i.e. less than 90 calculations) as shown in Figure 18, the additional cost (i.e. 36 extra calculations) being only necessary for a more accurate prediction of the maximum real part of eigenvalues as indicated in Figure 17.

In conclusion the use of a GA enables to identify with a few CEA the area with the maximum real part and the most influential contact states are quickly identified for different frequency range on interest. Actually, it is the accurate determination of the maximum real part that requires a higher number of computations. In a context were the case scenario defined by "the most unstable configuration" could be determined with a margin of error, the GA gives satisfactory results. The characteristics of the most unstable configuration are estimated by running only 86 CEA instead of the 512 calculations to be done without GA. So this strategy appears to be of interest for an industrial point of view in order to find the most harmful design in regard to the internal contact interfaces while saving in numerical computing time.

\section{Conclusion}

The objective of the present study was to investigate the influence of internal contacts of a disc brake system on its squeal noise propensity. Indeed, the caliper, the bracket, the piston and the pads can interact through contact interfaces and so, influence the stability of the system which is determined with the CEA. In order to work with affordable computational time, the different contacts are modeled by two different states: frictional or not. Then, this numerical study reveals that they have a strong impact on the squeal propensity. A deeper analyze establishes the sensitivity of the unstable modes to these contact formulation and prevents their use as a criterion to track instabilities. It insights the difficulty to predict all instabilities with a unique configuration and highlights the need to consider various configurations in order to improve the prediction of squeal noise. However, the consideration of all contact conditions during the design process of a brake system is a necessity but it is nowadays unaffordable because of the prohibitive number of calculations to perform. To cope with this difficulty, the present study proposes a methodology to rank the role of internal contacts and to determine the most influential of them. The study here demonstrated that a GA has the ability to quickly identify the most influential contacts and the worst-case scenario.

The important role of the internal contact conditions was demonstrated. The high number of potential cases to be studied by considering various internal contact conditions can be time consuming. Hence only two different contact conditions were retained in order to reduce the size of the problem and hence to save computational times. Then, the proposed strategy consists to find the worst-case scenario in term of the "most unstable configuration" (i.e. the configuration with the highest value of real parts) and to apply the design process on this case. However, it could be conceivable to consider uncertainties on the contact conditions in order to realize a robust design process. If this strategy is probably the most adapted to the problem, the consideration of this nine additional parameters in the design process is still a scientific barrier.

Although this study points out an additional difficulty in predicting squeal noise and proposes a strategy for dealing with it in a limited number of calculations, some interesting developments should be considered. First, the strategy adopted here to predict the squeal propensity is based on the CEA, which may lead to an under- or over-estimation of unstable modes. It might be interesting to see if the influence on the dynamic of the system is as important. Moreover, the worst-case scenario presented here is based on only one criterion, namely the 


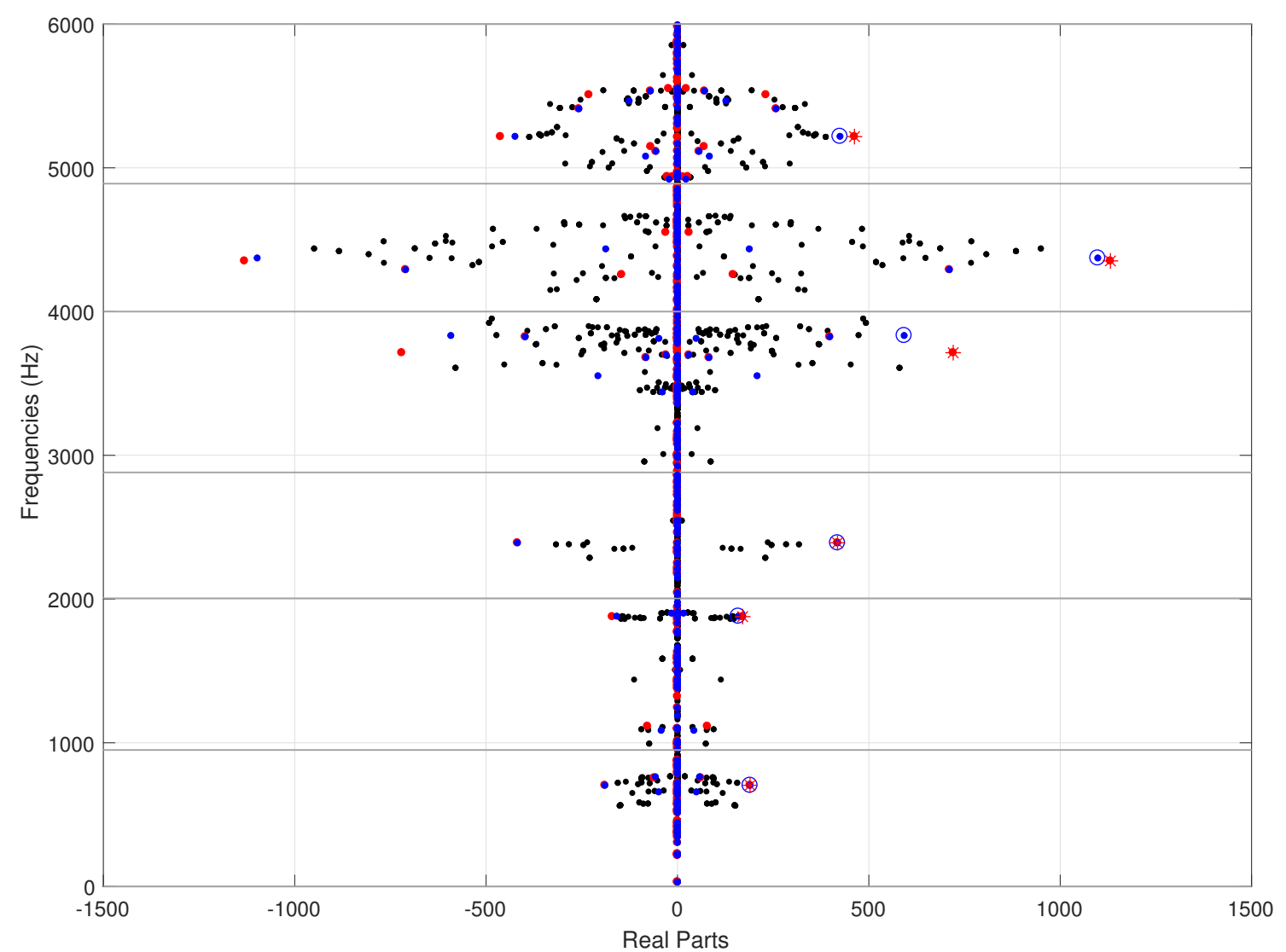

(a)

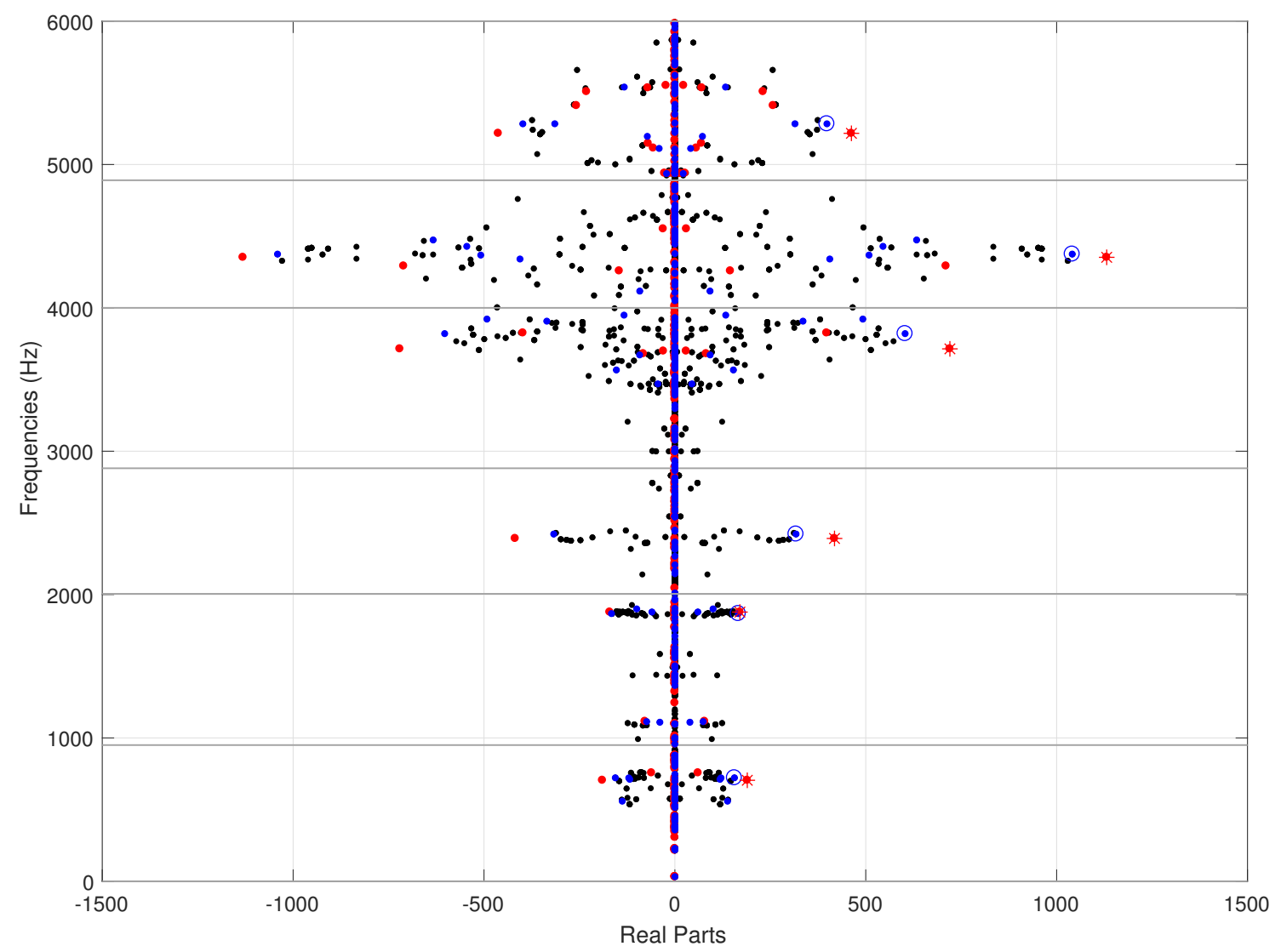

(b)

Figure 16: Eigenvalues computed during the algorithm with the first (a) and second (b) sets of parameters: black: eigenvalues computed during the algorithm, red stars: theoretical solutions, red points: eigenvalues of the theoretical solutions, blue circle: solutions of the algorithm 


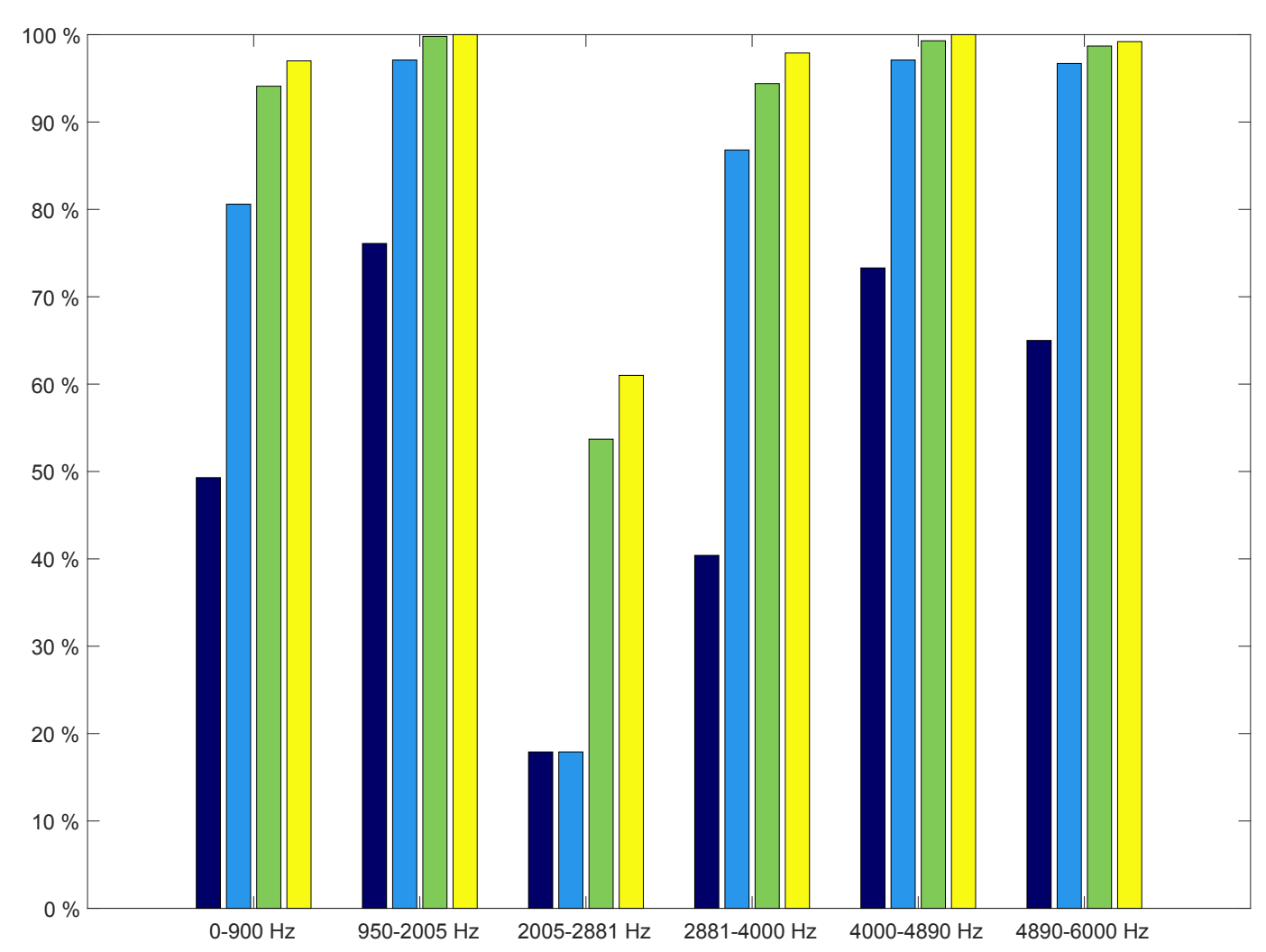

(a)

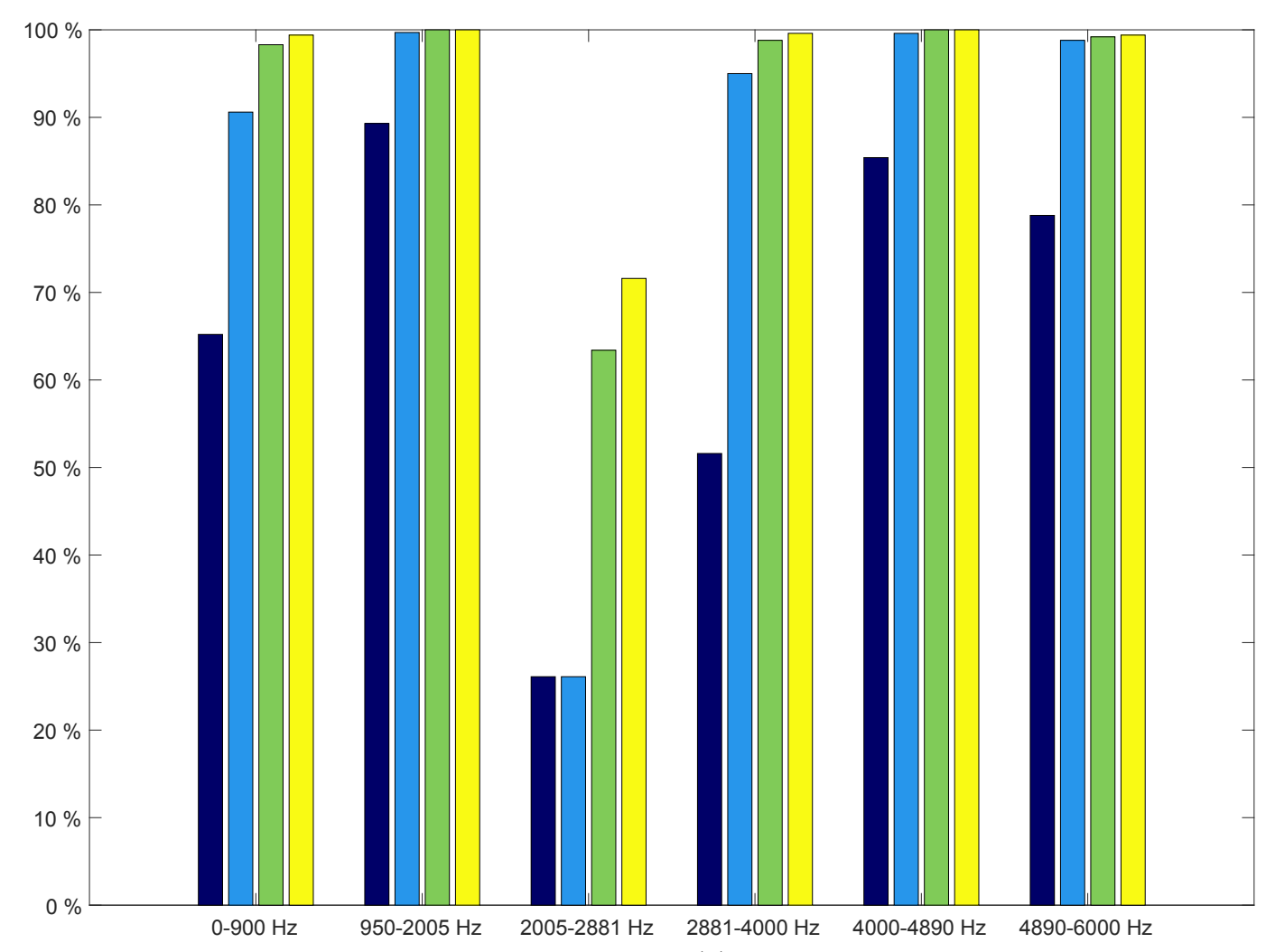

(b)

Figure 17: Precision of the solution obtained for 1000 initializations for the first (a) and second (b) set of parameters on each frequency range - dark blue: $10 \%$ of error, blue: $20 \%$ of error, green: $25 \%$ of error and yellow: $30 \%$ of error 

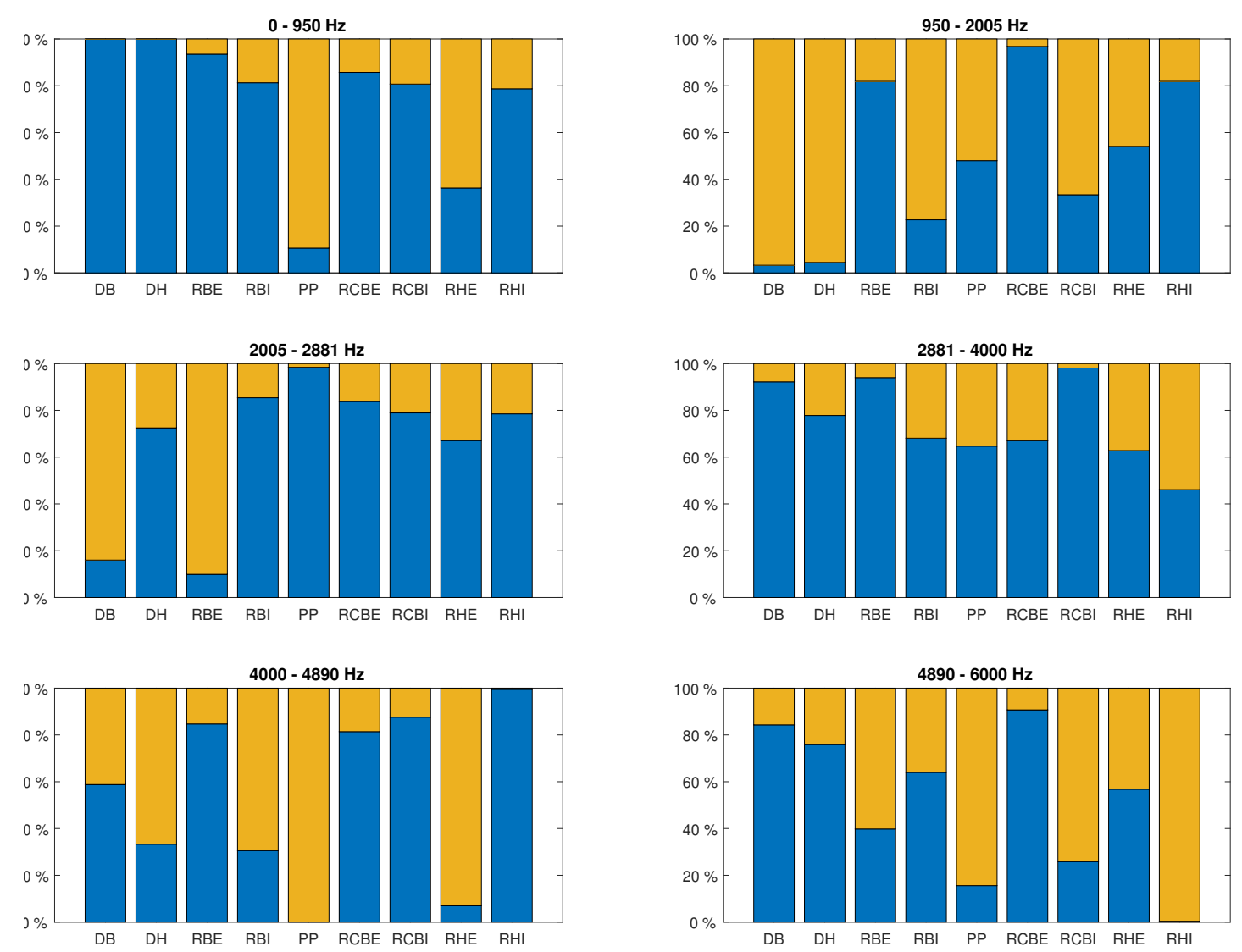

(a)
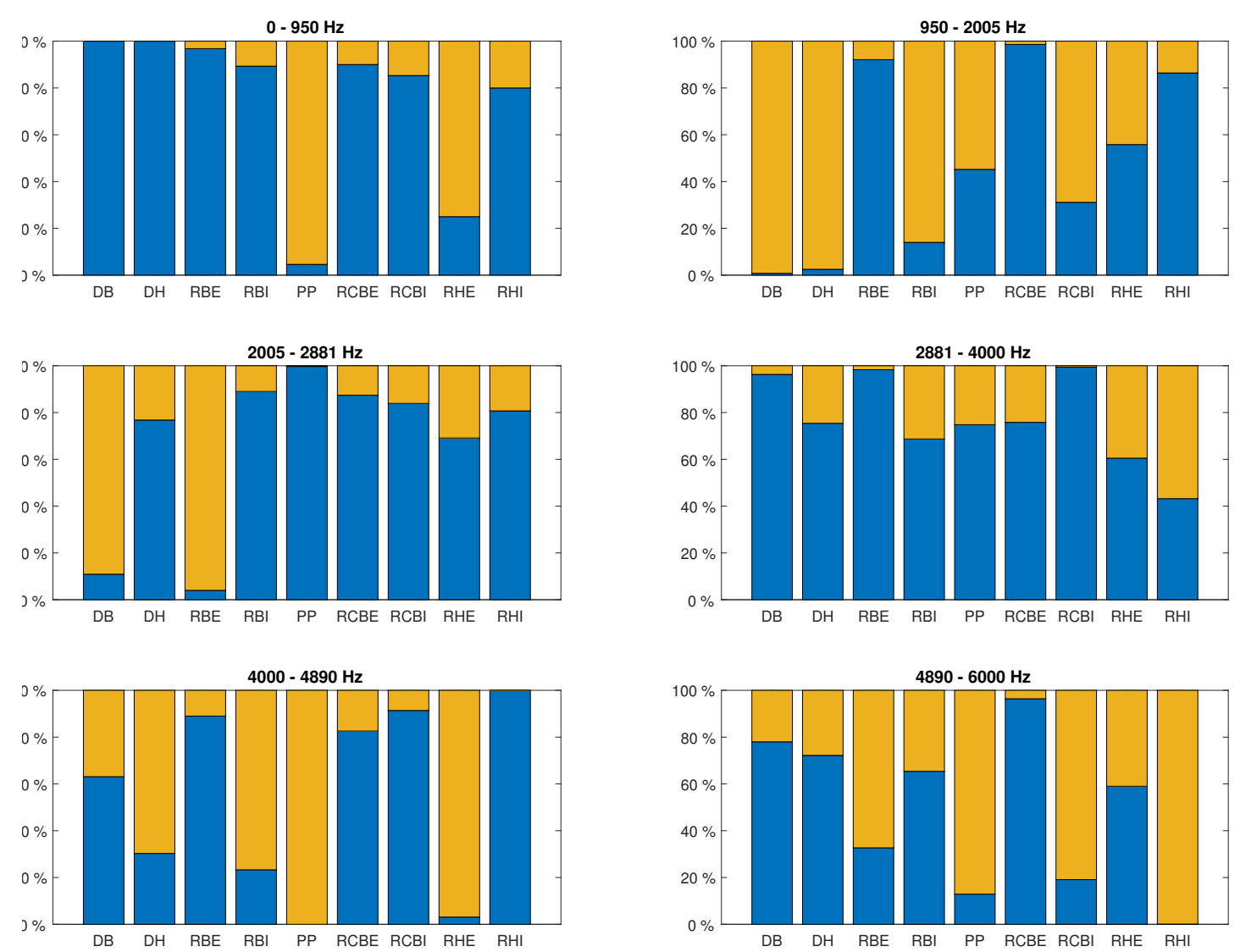

(b)

Figure 18: Repartition of the contact states of the solution obtained for 1000 initializations for the first (a) and the second (b) sets of parameters on each frequency range - Blue: non-frictional state and yellow: frictional state 
highest growth rate, it might be interesting to investigate another criterion. Last but not least, one of the most crucial next steps is to study the squeal propensity, with all contact conditions, of the brake after design on the worst-case to verify that the reduction of the level of instability on the most unstable configuration actually implies a reduction of the level of instability on all configurations.

\section{Acknowledgments}

This work was achieved within PSA Peugeot Citroën Stellab program - OpenLab Vibro-Acoustic- Tribology@Lyon (VAT@Lyon).

J.-J. Sinou acknowledges the support of the Institut Universitaire de France.

\section{References}

[1] O. Fazio, S. Nacivet, and J-J. Sinou. Reduction strategy for a brake system with local frictional non-linearities - application for the prediction of unstable vibration modes. Applied Acoustics, 91:12-24, 2015.

[2] T. Tison, A. Heussaff, F. Massa, I. Turpin, and R.F. Nunes. Improvement in the predictivity of squeal simulations: Uncertainty and robustness. Journal of Sound and Vibration, 333:3394 - 3412, 2014.

[3] A. Nobari, H. Ouyang, and P. Bannister. Uncertainty quantification of squeal instability via surrogate modelling. Mechanical Systems and Signal Processing, 60-61:887 - 908, 2015.

[4] Y. Dai and T.C. Lim. Suppression of brake squeal noise applying finite element brake and pad model enhanced by spectralbased assurance criteria. Applied Acoustics, 69:196 - 214, 2008.

[5] H. Lü, W.-B. Shangguan, and D. Yu. A unified approach for squeal instability analysis of disc brakes with two types of random-fuzzy uncertainties. Mechanical Systems and Signal Processing, 93:281 - 298, 2017.

[6] S. Oberst and J.C.S. Lai. Nonlinear transient and chaotic interactions in disc brake squeal. Journal of Sound and Vibration, $342: 272-289,2015$.

[7] H. Festjens, G. Chevallier, F. Renaud, J.-L. Dion, and R. Lemaire. Effectiveness of multilayer viscoelastic insulators to prevent occurrences of brake squeal: A numerical study. Applied Acoustics, 73:1121 - 1128, 2012.

[8] M. Treimer, B. Allert, K. Dylla, and G. Müller. Uncertainty quantification applied to the mode coupling phenomenon. Journal of Sound and Vibration, 388:171 - 187, 2017.

[9] A. R. AbuBakar and H. Ouyang. Complex eigenvalue analysis and dynamic transient analysis in predicting disc brake squeal. International Journal of Vehicle Noise and Vibration, 2:143-155, 2006.

[10] F. Massi, L. Baillet, O. Giannini, and A. Sestieri. Linear and non-linear numerical approaches. Mechanical Systems and Signal Processing, 21:2374-2393, 2007.

[11] A. Loyer, J-J. Sinou, O. Chiello, and X. Lorang. Study of nonlinear behaviors and modal reductions for friction destabilized systems. application to an elastic layer. Journal of Sound and Vibration, 331:1011-1041, 2012.

[12] R.A. Ibrahim. Friction-induced vibration, chatter, squeal and chaos part ii: Dynamics and modelling. Applied Mechanics Review, 47:227-253, 1994.

[13] R.A. Ibrahim. Friction-induced vibration, chatter, squeal and chaos part 1: mechanics of contact and friction. Applied Mechanics Review, 47:209-226, 1994.

[14] D.A. Crolla and A.M. Lang. Brake noise and vibration - state of art. Vehicule Tribology, Tribology Series, 18:165-174, 1991.

[15] R.T. Spurr. A theory of brake squeal. Proceedings of the Automobile Division, Institution of Mechanical Engineers, 1:33-40, 1961.

[16] N.M. Kindkaid, O.M. O'Reilly, and P. Papadopoulos. Automative disc brake squeal. Journal of Sound and Vibration, 267:105-166, 2003.

[17] H. Ouyang, W. Nack, Y. Yuan, and F. Chen. Numerical analysis of automative disc brake squeal: a review. International Journal of Vehicle Noise and Vibration, 1:207-231, 2005.

[18] A. Papinniemi, J.C.S Lai, J. hao, and L. Loader. Brake squeal: a literature review. Applied Acoustics, 63:391-400, 2002.

[19] E. Sarrouy, O. Dessombz, and J-J. Sinou. Piecewise polynomial chaos expansion with an application to brake squeal of a linear brake system. Journal of Sound and Vibration, 332(3):577-594, 2013.

[20] L. Nechak, F. Gillot, S. Besset, and J-J. Sinou. Sensitivity analysis and kriging based models for robust stability analysis of brake systems. Mechanics Research Communications, 69:136-145, 2015.

[21] H. Lü and D. Yu. Brake squeal reduction of vehicle disc brake system with interval parameters by uncertain optimization. Journal of Sound and Vibration, 333(26):7313-7325, 2014.

[22] H.Q. Do, F. Massa, T. Tison, and B. Lallemand. A global strategy for the stability analysis of friction induced vibration problem with parameter variations. Mechanical Systems and Signal Processing, 84:346-364, 2017.

[23] S. Nacivet and J-J. Sinou. Modal amplitude stability analysis and its application to brake squeal. Applied Acoustics, 116:127$138,2017$.

[24] K. Soobbarayen, J-J. Sinou, and S. Besset. Numerical study of friction-induced instability and acoustic radiation-effect of ramp loading on the squeal propensity for a simplified brake model. Journal of Sound and Vibration, 333(21):5475-5493, 2014.

[25] K. Soobbarayen, S. Besset, and J-J. Sinou. A simplified approach for the calculation of acoustic emission in the case of friction-induced noise and vibration. Mechanical Systems and Signal Processing, 50:732-756, 2015.

[26] S. Oberst, J.C.S. Lai, and S. Marburg. Guidelines for numerical vibration and acoustic analysis of disc brake squeal using simple models of brake systems. Journal of Sound and Vibration, 332(9):2284-2299, 2013.

[27] J-J. Sinou, A. Loyer, O. Chiello, G. Mogenier, X. Lorang, F. Cocheteux, and S. Bellaj. A global strategy based on experiments and simulations for squeal prediction on industrial railway brakes. Journal of Sound and Vibration, 332(20):5068-5085, 2013.

[28] G. Fritz, J-J. Sinou, J-M. Duffal, and L. Jézéquel. Investigation of the relationship between damping and mode-coupling patterns in case of brake squeal. Journal of Sound and Vibration, 307(3-5):591-609, 2007.

[29] G. Fritz, J-J. Sinou, J-M. Duffal, and L. Jézéquel. Effects of damping on brake squeal coalescence patterns-application on a finite element model. Mechanics Research Communications, 34(2):181-190, 2007. 
[30] O.N. Kirillov and A.O. Seyranian. The effect of small internal and external damping on the stability of distributed nonconservative systems. Journal of Applied Mathematics and Mechanics, 69(4):529-552, 2005.

[31] K. Deb, A. Pratap, S. Agarwal, and T. Meyarivan. A fast and elitist multiobjective genetic algorithm: Nsga-ii. IEEE transactions on evolutionary computation, 6(2):182-197, 2002.

[32] D. Whitley. A genetic algorithm tutorial. Statistics and computing, 4(2):65-85, 1994. 